\title{
Review Article \\ Building Spinal and Brain Commissures: Axon Guidance at the Midline
}

\author{
Valérie Castellani \\ University of Lyon, University Claude Bernard Lyon1, CGphiMC UMR CNRS 5534, 16 rue Raphael Dubois, 69000 Lyon, France
}

Correspondence should be addressed to Valérie Castellani; valerie.castellani@univ-lyonl.fr

Received 3 April 2013; Accepted 23 April 2013

Academic Editors: V. M. Golubovskaya, Z. Pan, W. K. Song, and T. Yazawa

Copyright (C) 2013 Valérie Castellani. This is an open access article distributed under the Creative Commons Attribution License, which permits unrestricted use, distribution, and reproduction in any medium, provided the original work is properly cited.

Commissural circuits are brain and spinal cord connections which interconnect the two sides of the central nervous system (CNS). They play essential roles in brain and spinal cord processing, ensuring left-right coordination and synchronization of information and commands. During the formation of neuronal circuits, all commissural neurons of the central nervous system must accomplish a common task, which is to project their axon onto the other side of the nervous system, across the midline that delineates the two halves of the CNS. How this task is accomplished has been the topic of extensive studies over the last past 20 years and remains one of the best models to investigate axon guidance mechanisms. In the first part of this review, I will introduce the commissural circuits, their general role in the physiology of the nervous system, and their recognized or suspected pathogenic properties in human diseases. In the second part of the review, I will concentrate on two commissural circuits, the spinal commissures and the corpus callosum, to detail the cellular and molecular mechanisms governing their formation, mostly during their navigation at the midline.

\section{Introduction}

1.1. The Commissural Circuitry. The commissural circuits form a complex network of projections interconnecting both sides of the CNS of bilaterals. Commissural neurons are present at all levels of the CNS and extend their axon across the midline to build circuits that are crucial for the integration of sensory modalities, motor commands, and processing of brain functions [1]. The telencephalon elaborates three major commissures: the anterior commissure, the hippocampal commissure, and the corpus callosum. The anterior commissure is composed of two branches interconnecting the temporal lobes and is implicated in emotional processing. The hippocampal commissure interconnects the left and right hippocampus, an enrolled gyrus flanking the lateral ventricle at temporal levels. The corpus callosum, which is in humans the largest tract of the brain, emerged in placental mammals [2]. It radiates dorsally from the whole cerebral cortex to interconnect left and right cortical areas (Figure 1(a)). Other brain structures elaborate additional commissures, such as the posterior commissure, which interconnects nuclei of the diencephalon. Finally, several axon tracts navigating long distances cross the midline at various places of the CNS. Organisms with bilateral vision have crossed and uncrossed visual pathways. The visual fibers arising from each eye cross the midline ventrally at the optic chiasm and auditory fibers at the level of the pons. Corticospinal projections contributing to voluntary movement and fine motility descend ventrally from the cerebral cortex and decussate at the medulla/spinal cord boundary, this midline crossing being coupled with a ventral to dorsal change of navigation. In the spinal cord, the coordination of left/right motor behaviors essential for alternate and synchronized activities is ensured by spinal commissural projections, which course and cross the midline at ventral position to convey information towards higher brain centers (Figure 1(b)).

1.2. Commissural Circuits and Neurodevelopmental Pathologies. Defective navigation of commissural projections has been observed in various types of neurodevelopmental pathologies and in diseases suspected to originate from early alterations in the construction of neuronal circuits. Corpus callosum agenesis is one of the most frequent congenital 


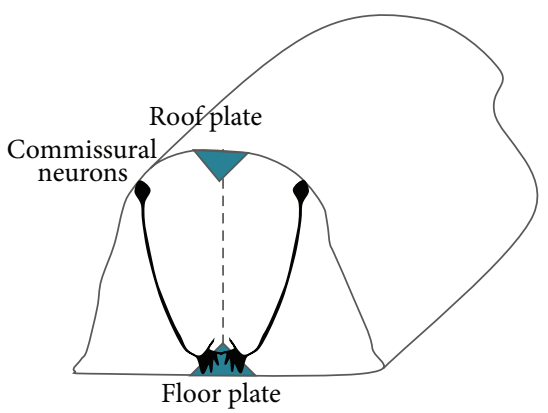

(a)

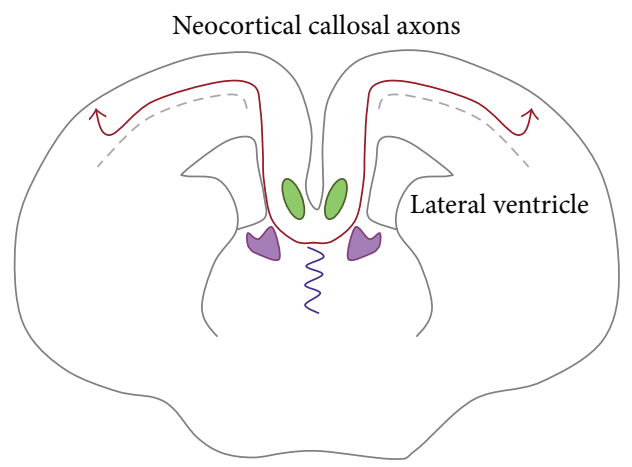

(b)

FIGURE 1: Schematic representation of the spinal commissural and corpus callosum formation. (a) Callosal axons arise from neurons of the cerebral cortex, which turn medially and grow across the dorsal midline of the telencephalon. (b) Dorsally located interneurons of the left and right hemispinal cord extend axons which navigate towards the ventral floor plate in which they cross the midline. After crossing, commissural axons turn rostrally and navigate parallel to the floor plate to reach their target cells.

malformations, present in 3 to $7 \%$ of 1000 births [3]. Corpus callosum agenesis and hypoplasia (partial agenesis) are associated with various developmental syndromes, such as Andermann, Apert, X-linked hydrocephalia (also referred to as MASA syndrome). Mutations in at least thirty genes have been characterized from genetic analysis of patients carrying corpus callosum agenesis [4]. Some of these mutations such as those in the L1CAM gene causing mental retardation also affect the formation of the corticospinal tract, resulting in additional functional impairment such as thumb abduction [5]. Mutations affecting midline crossing of the CST also result in mirror movement synkinesis. This motor disorder, where patients when commanding the movement of one hand or forearm move also in mirror the other one simultaneously, is associated with several syndromes $[1,6]$. For example up to $75 \%$ of patients with Klippel-Feil syndrome present mirror movement synkinesis and $85 \%$ for the X-linked form of the Kallmann syndrome.

In contrast to the corticospinal tract and the corpus callosum, whose malformations have been well correlated to developmental human pathologies, much less is known on the consequences of anterior commissure defects. However, defect of anterior commissure is reported in a number of neurodevelopmental diseases. Guided by the implication of the anterior commissure in emotional processing, Saxena and collaborators found reduction of the anterior commissure thickness in children with aggressive bipolar disorders [7]. Schizophrenic patients and particularly the youngest ones were reported to exhibit reduced interhemispheric connectivity, and this appeared to also concern the anterior commissure, as indicated by quantitative analysis of the commissure thickness using diffusion tensor imaging [8]. The anterior commissure thickness was also investigated in patients with chiari II malformation, a congenital disease with several brain abnormalities [9], and in contrast to the previous examples was found significantly increased [10]. A study also reported rudimentary anterior commissure and hypoplasia of several other commissural midline structures, in a case of Cornelia de Lange Syndrome [11], a multisystem developmental disorder characterized by growth retardation, cognitive impairment, structural malformations, and characteristic facial features [12]. Missense mutations in a gene encoding Tubb3, a neuron-specific type of tubulin, were found to cause neurological disorders associating abnormal development of brain commissures including the anterior commissure [13]. Investigation of children carrying mutations in the Pax6 gene and suffering from congenital aniridia, a disorder characterized by the absence of iris but also multiple alterations of optic structures, associating in some cases cerebellar ataxia and mental retardation [14], showed reduction of the anterior commissure in $70 \%$ of cases [15]. Recent literature also attributes an important function of the interhemispheric connectivity including the anterior commissure to language acquisition and alterations in language dysfunctions [16]. Despite this literature, very little is yet known concerning the pathogenic processes compromising the development of these commissural circuits. Nevertheless, advances in genetics and neuroimaging clearly sparked a growing interest for axon guidance mechanisms and the potential pathogenic consequences of their alterations.

1.3. General Mechanisms of Axon Navigation. The famous neuroanatomist Santiago Ramon y Cajal discovered at the end of the nineteenth century that navigating axons are equipped with a terminal enlarged structure, the growth cone. It is composed of a central domain filled with microtubules and a peripheral domain of actin-rich filopodia and lamellipodia (Figure 2(a)). These peripheral structures extend and retract, exploring the environment and sensing extracellular cues [17-19]. Neurons expose a variety of receptors at the growth cone surface that allow them to respond to the corresponding ligands. These ligands have a particular spatial distribution in the environment and thus by providing an attractive or a repulsive message can orient the axons in a given direction (Figure 2(b)). Activation of the guidance receptors by the ligands is the first step of a series of signaling cascades leading to the remodeling of the growth cone cytoskeleton, actin and microtubules. This results in the generation of asymmetry of the peripheral growth cone structures reflecting the spatial distribution of the ligand, thus triggering the steering of the growth cone away or towards 


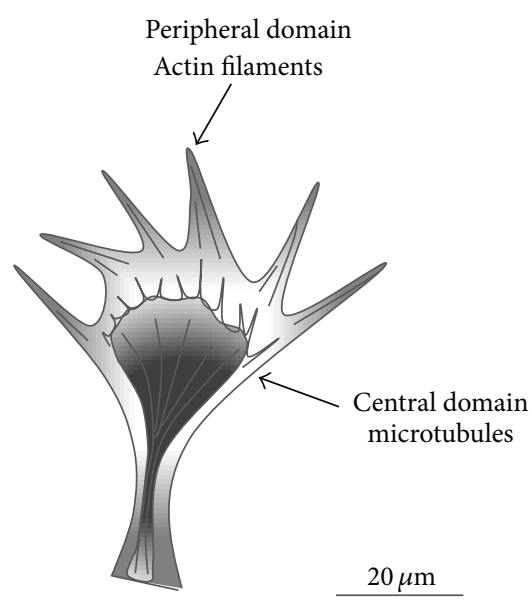

(a)

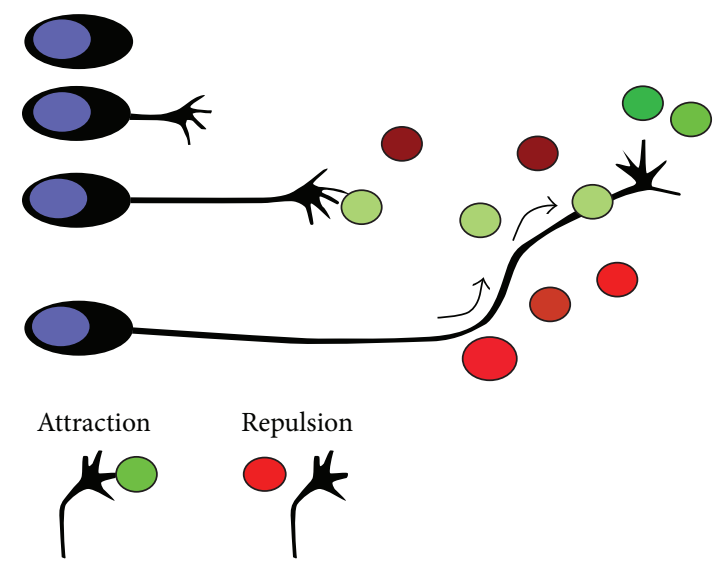

(b)

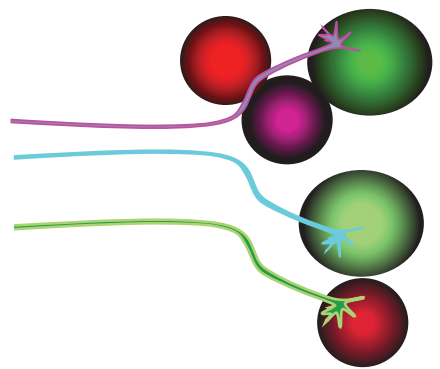

(c)

Figure 2: Structure and function of the growth cone in axon navigation. (a) The growth cone that tips the axon is a highly motile structure composed of a microtubule-rich central domain and an actin-rich peripheral domain, forming lamellipodia and filopodia. During axon navigation, the growth cone explores its environment by extending and retracting its filopodia. (b) The axon trajectory is defined by environmental cues which exert attractive (in green) and repulsive (in red) effects on the growth cone. (c) Axons from different neuron types (here in blue, pink, and green) express specific combinations of receptors and thus respond differently to the same cues present in their environment and thus navigating distinct pathways.

the ligand source, depending on its repulsive or attractive nature [19]. An increasing number of protein families have been found to be implicated in the guidance of neuronal projections, among which are the classical Semaphorins, Netrins, Slits and Ephrins and their corresponding receptors Plexins/Neuropilins, DCC, Robos, and Ephs, respectively. Extracellular cues acting as morphogens in early stages of the embryonic development were also found in the recent years to play a role in the context of axon and cell migration. This is the case for Shh, Wnts, and BMPs [18].

The specific growth cone equipment of guidance receptors together with the specific distribution of guidance ligands sets a first level of selectivity in axon tract trajectories (Figure 2(c)). Nevertheless, genes encoding guidance receptors and ligands would not be in sufficient numbers to achieve the complex network of trajectories needed to build all neuronal circuits of an organism, even those of the simplest ones. Thus, additional mechanisms must take place to generate further diversity from this rather limited set of cues. The navigation of commissural axons and particularly the key step of midline crossing, provided crucial insights into this question. Extensive investigations in recent years revealed an extreme degree of complexity, with series of steps implicating modulation of axon responsiveness to the various guidance cues orienting axon trajectories towards, within, and away from the midline. The emerging key message resides in the central role of processes controlling the spatial and temporal fine tuning of the guidance molecular machinery over midline crossing. These mechanisms will be discussed for the two model systems described in this review, the spinal commissural projections and the corpus callosum.

\section{Navigation of Spinal Commissural Axons}

During locomotion, the left and right sides of the body must be coordinated to ensure proper alternation or synchrony of muscle activities, depending on the nature of the movement. This is achieved by the commissural circuits of interneurons of the spinal cord. Several populations of interneurons settle at dorsal levels, which are subdivided into early-born neurons forming the classes dI1 to dI6 and late-born neurons composing the dILA and dILB groups [20]. The proprioceptive dll class settles at the most dorsal position of the spinal cord, close to the roof plate, and transmits information 
to the cerebellum via the spinocerebellar and the cuneocerebellar tracts. It is composed of two types of neurons, those establishing ipsilateral projections (dlli) and those establishing commissural projections (dl1c) [21].

\subsection{Genetic Program Specifying Commissural versus Ipsilateral} Trajectories. Combinations of transcription factors specify these different lineages of the spinal cord [22]. Morphogens of the TGF $\beta$ and Wnt families produced by the roof plate act upstream in the specification of some of these lineages, including the dll population $[23,24]$. Dll neurons are generated from a pool of progenitor cells expressing Mathl, and can be identified by two LIM transcription factors, Lhx2 and Lhx9 [21, 22]. Evidence from mouse genetics indicates that the developmental programs assigning cell identity also specify the commissural versus ipsilateral nature of the projections. Wilson and collaborators analyzed axonal patterns in mice expressing a fluorescent reporter under the control of Mathl and Barhl2 promotors, specific to dl1 neurons [25]. Interestingly, the temporal Lhx 2 and Lhx9 expression allows dll ipsilateral neurons to be distinguished from commissural neurons. At early stages during neuron migration from the proliferative zone of the spinal cord, close to the central canal, Lhx2 and Lhx 9 are strongly expressed by all neurons. Later, Lhx2 and Lhx9 are downregulated in commissural neurons, while Lhx2 is retained in ipsilateral neurons. In mice lacking $\operatorname{Lhx} 2$ and $\operatorname{Lh} x 9$, all dll projections are ipsilateral, while invalidation of one of the genes is not sufficient to significantly abrogate the commissural circuit. Thus, Lhx2 and Lhx9 instruct commissural neurons to send their axon across the midline. Conversely, the nature of the transcription factors specifying ipsilateral projection patterns remained unclear until recently. Ding and collaborators investigated the role of the mammalian BAR class transcription factors, Barhll and Barhl2, in the specification of contralateral and ipsilateral projection pattern of dll1 neurons [26]. Analysis of the lacZ reporter in Barhl2-lacZ knock-in mice with markers of the different populations of dorsal interneurons indicated that both genes are found expressed in postmitotic dlli and dllc neurons and not in the dl2-6 populations. Despite an apparent lack of specific expression in the ipsilateral or contralateral subdivision of the dll1 pool, the deletion of Barhl2 in mice strongly diminished the proportion of dll1 neurons with ipsilateral projections. dlli (ipsilateral) and dllc (commissural) neurons can be distinguished by their distinct position in the dorsal horn, residing in the lateral and medial deep dorsal horn, respectively. Deletion of Barhl2 resulted in depletion of neurons in the lateral deep dorsal horn, where the dlli group should normally be. Moreover, Barhl2 loss also resulted in increase of Lhx 2 expression in Dl1 neurons, normally downregulated in the dllc group. Altogether these data provided evidence that loss of Barhl2 induced dllii neurons to adopt a dlic fate, thus switching their projection pattern from ipsilateral to contralateral [26].

In the recent years, other key molecular determinants of the ipsilateral feature of the projections have been identified in the visual system. In organisms with binocular vision, each retina conveys the visual information to nervous centers located in both sides of the brain [27]. Ganglion cell neurons with different identities settle at different positions in the retina and establish commissural or ipsilateral projections, thus crossing or not crossing the midline at the optic chiasm. Garcia-Frigola and colleagues demonstrated that the transcription factor Zic2 is necessary and sufficient to induce an ipsilateral pathway at the optic chiasm. The authors showed by electroporation of mouse embryos that ectopically expressing Zic2 in neurons normally projecting contralaterally resulted in an ipsilateral pathway of their axons.

In the next paragraph, I will review the mechanisms controlling the navigation of commissural axons, with three essential steps: navigation towards the midline, across the midline, and after midline crossing. The principal guidance cues acting during these steps are summarized in Figure 3.

\subsection{Navigation of Commissural Axons: Step 1: From Dorsal to Ventral}

2.2.1. Action of Dorsal Chemorepellents. Once the neuron is assigned with a projection pattern, the trajectory of its axon will be controlled at all steps of the navigation, with a battery of mechanisms. First, the axon of the dll neuron is oriented towards the ventral side of the spinal cord. Evidence that a guidance force acts to drive the direction of the initial growth of commissural axons came from clever experiments in which the investigators evidenced the existence of chemorepulsive signals emanating from the roof plate and identified them as the Bone Morphogenetic Proteins (BMPs) BMP7 and the Growth Differentiation factor GDF7 [28]. To demonstrate this, the authors designed a coculture in which a piece of roof plate tissue was placed adjacent to a spinal cord open book. The authors could show that the tissue deflected away the commissural axons, and this effect could be mimicked by a cell aggregate secreting BMP7 [28]. In the recent years, the Butler lab extended its observations, showing that BMPs influence the rate of commissural axon extension towards the floor plate [29]. Perturbation of axon growth rates might have deleterious consequences as timing guidance cue expression profiles with the arrival of axons is essential for proper axon pathfinding. Many guidance cues have been observed in in vitro setup to regulate the trajectory of axons but also their elongation per se [30]. Thus to which extent the delay of axon arrival at choice points due to alterations of the axon growth rate causes guidance errors in vivo is an interesting issue that should be further assessed.

More recently, a second repulsive signal emanating from the roof plate has been identified, named Draxin [31]. This novel axon guidance molecule exhibited a strong inhibitory effect on axon growth from dorsal spinal cord explants. In cocultures, cell aggregates secreting Draxin deflected away the axons extending from the explants. Draxin overexpression in the chick neural tube disrupted the navigation of commissural axons. In mice lacking Draxin, the commissural tracts still coursed towards the floor plate but were defasciculated, a phenotype that is consistent with decrease of environmental repulsive forces. Thus, the initial trajectory of commissural 


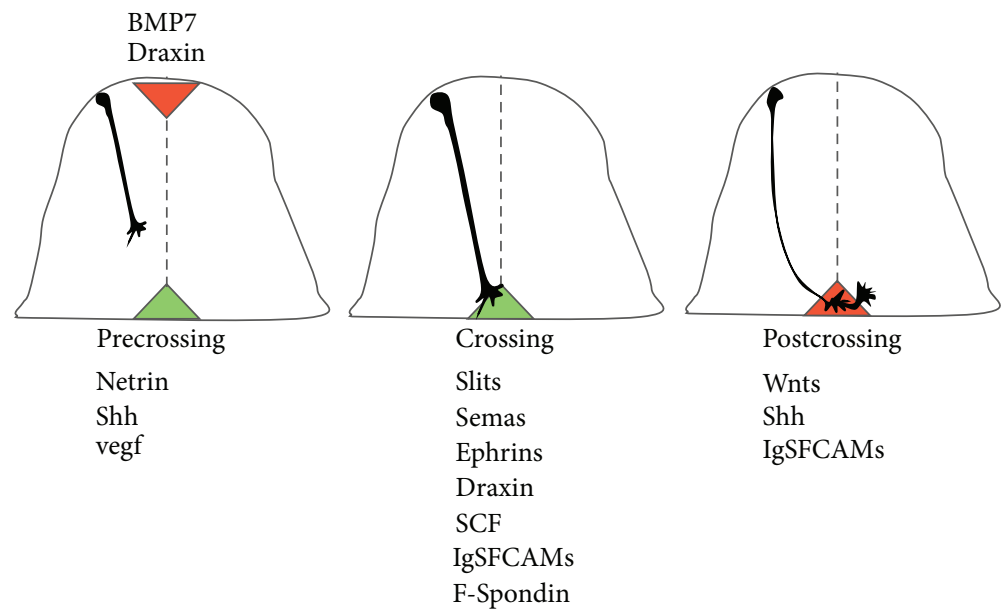

FIGURE 3: Guidance of spinal commissural projections. The scheme illustrates the different steps of commissural axon navigation in the spinal and the set of guidance molecules acting as attractants and repellents for the axons.

axons appears mainly to be controlled by a set of chemorepellents emanating from adjacent dorsal territories.

2.2.2. Action of Ventral Chemoattractants. When growing towards the ventral side of the spinal cord, the axons are maintained in compact bundles. When reaching the ventral side, they start perceiving guidance signals emanating from a key intermediate target along their pathway, the floor plate, that they must enter to cross the midline. The development of ex vivo models in which commissural tissue was cocultured at a distance from pieces of floor plate in three-dimensional gels played a key role in the investigation of commissural axon navigation. Netrinl was the first guidance molecule identified by the Tessier-Lavigne lab as a floor-plate-derived chemoattractant for commissural neurons [32], activating the receptor DCC to achieve this effect (Deleted in colorectal cancer; $[33,34])$. Netrin1 mRNA is detected in the floor plate of chick and mouse spinal cords at the appropriate period during the navigation of commissural axons, and forms a high ventral to low dorsal gradient. Immunodetection of Netrinl in embryonic sections supports that the protein might diffuse from the ventral source [35]. In flies, Netrin1 is also expressed at the ventral midline and might act over short distances since preventing its secretion by anchoring the protein at the membrane does not impact on the formation of the commissures [36]. A second Netrin gene has been found in the vertebrate and invertebrate (fly) genome, encoding a protein, Netrin2, which shares Netrin1 function during commissural axon guidance [32, 37]. Although DCC is assumed to be the principal mediator of Netrin attraction in commissural axons, other proteins have been identified as Netrin receptors during commissural axon guidance. The down syndrome cell adhesion molecule (DSCAM) was shown to bind Netrinl and in ovo knockdown with RNAi disturbed commissural axon guidance $[38,39]$. Nevertheless, spinal commissures were observed to form normally in mice lacking DSCAM, indicating that DSCAM is dispensable in the navigation of commissural axons towards the floor plate
[40]. Early work by Corset and collaborators reported that the $G$ protein-coupled adenosine receptor A2b binds Netrin and can mediate Netrin-induced axon outgrowth of commissural neurons [41]. Additional investigation of the A2b receptor reported that it might act indirectly: its activation induces removal of another Netrin receptor that mediates repulsion, Unc5A, from the cell surface, thus eliciting an attractive growth cone response [42]. Recent work showed that the amyloid precursor protein (APP) interacts with DCC and acts as coreceptor for Netrin-mediated guidance of spinal commissural axons [43]. Thus, main and accessory Netrin receptors appear to participate in the guidance of spinal commissural axons towards the floor plate. Their exact and respective contribution still remains unclear and is one of the challenges in the field.

Shh via Boc receptor and more recently VEGF via Flk1 receptor were shown to act in synergy with Netrins, providing a strong attraction towards the floor plate. Accordingly, deletion in mice of each or several of these chemoattractants affected more or less profoundly the ability of commissural axons to reach the floor plate $[33,34,44-46]$.

2.3. Navigation of Commissural Axons: Step 2: Navigating the Floor Plate. The floor plate is a transient structure formed by a small group of ependymal cells at the border of the ventral canal. As its dorsal roof plate counterpart, it has many key developmental functions [47]. First at early embryonic stages, it acts as an organizing center, producing the morphogen Shh, which patterns cell identities of the neural tube [48]. Second, the floor plate has the capacity to generate large numbers of neurons, although this is true for the midbrain but not for the hindbrain and the spinal cord, highlighting the heterogeneity of floor plate cells along the neuraxis [49]. Third, the floor plate segregates the ipsilateral and contralateral axon projections. It acts from the onset as a barrier for ipsilateral axons, preventing them from crossing. In contrast, this barrier function only operates for contralateral axons after they have crossed so that they are allowed to cross 
the midline once. To achieve this barrier function, the floor plate expresses guidance cues that are interpreted in distinct ways by ipsilateral and contralateral growth cones. Moreover, floor plate cells also secrete cues that modify the sensitivity of commissural growth cones to these local guidance cues and thus organize the crossing of the axons [50-52].

The processes underlying the barrier function of the floor plate rely on the temporally controlled and differential sensitivity of commissural and ipsilateral axons to floor plate repellents that belong to the Slit, Ephrin, and Semaphorin families [53-56]. The current view essentially based on studies of the Robo/Slit signaling is that ipsilateral axons are sensitive from the onset to the repellents and this prevents their crossing. Contralateral axons are initially insensitive to the repellents so they can cross the midline. During crossing, they acquire sensitivity to the repellents so they are prevented from recrossing the midline.

2.3.1. Gain of Response to Repellents. (1) The Robo-Slit Signaling. The role of the Robo/Slit signaling has been particularly well studied in the Drosophila model where glial cells at the midline of the ventral chord segregate ipsilateral and contralateral axons, as does the vertebrate floor plate. More than a decade ago, the Comm gene (Commissureless) was discovered through a genetic screen to control the ipsilateral and commissural choices of axons navigating the floor plate. Ipsilateral axons expressing Robo but not Comm perceive the Slit repellent and do not cross the midline. Experiments in which Comm was induced in ipsilateral neurons revealed that this turned their projections from ipsilateral to contralateral $[57,58]$. In contrast, Comm is transiently expressed in commissural neurons before midline crossing and sorts Robo to the late endocytic pathway, preventing the receptor from reaching the cell surface. In this way the sensitivity of precrossing commissural growth cones to Slit is silenced and allows midline crossing [59-63]. After midline crossing comm is downregulated. Consequently, Robo sorting at the growth cone surface is restored, establishing a sensitivity of commissural axons to Slit and switching on the barrier function of the floor plate [59, 64, 65]. Elucidating the mechanisms regulating Comm to better understand how Robo expression levels are controlled is thus one of the central issues in the field. It should be noted that Comm might not be the only regulator of Robo, or at least through this trafficking mechanism, as a recent study reported the presence of commissures in flies expressing a mutant Robo form escaping Comm-mediated sorting [66]. Moreover, the Robo2 gene could be implicated in a mechanism inhibiting Robol signaling. This possibility is supported by the finding that Commissures, which can be formed in flies with loss of function of Netrin signaling, are, however, completely absent when Robo2 is also abrogated [67].

Recent study by the Bashaw lab brought the first insights into how Comm is regulated in commissural neurons of the fly [68]. The investigators provided several lines of evidence supporting that Frazzled (Fra), the drosophila ortholog of the Netrin receptor DCC, is implicated in the regulation of Comm and this function is Netrin-independent. Loss of
Fra resulted in very little Comm expression in commissural neurons. Abrogation of Fra function using a dominant negative approach mimicked the functional consequences of Comm invalidation in commissural neurons, that is, a lack of commissures. Importantly, expressing Frain some ipsilateral neurons could induce ectopic Comm expression. Thus Fra is necessary and sufficient to induce Comm mRNA expression in commissural neurons. This, however, raises the question of how is Fra itself regulated. Liu and collaborators investigated the role of Midline (Mid) a member of the family of TBox transcription factors [69]. They provided evidence that in commissural neurons Mid exerts a direct transcriptional control on the Fra gene activation. The effect of Mid is not limited to Fra, as Robo and Slit were also found to be Mid transcriptional targets. Understanding the transcriptional regulations allowing Mid, Fra, and Comm to be specifically activated in commissural neurons and distinguishing neurons with ipsilateral and commissural projections is a key step towards elucidation of the mechanisms controlling the midline crossing choice.

In the vertebrate spinal cord, three Slits combine their effect to regulate commissural axon guidance at the midline, via Robol and Robo2. Analysis of single, double and triple mutant mouse lines highlighted the contribution of each Slit and brought the conclusion that they act in synergy [70] . The most notable difference is that the vertebrate genome lacks Comm. Nevertheless the temporal regulation of Commissural responsiveness to Slits between precrossing and postcrossing stages is conserved. Indeed, commissural axons exposed to a Slit source before crossing the floor plate do not show a repulsive response, whereas they do so after crossing [71]. Thus an exciting issue has been and remains to elucidate the mechanisms by which the sensitivity of commissural axons to Slits is regulated in vertebrates.

Important insights came from the study by the TessierLavigne lab of a third Robo gene, Robo3. Several slice variants are produced from the Robo3 locus. Interestingly, Chen and colleagues [72] reported that two Robo3 isoforms, generated from differential splicing of the $3^{\prime}$ end of the pre-mRNA and differed in their intracellular domain, have a temporal specific expression pattern in Commissural neurons. Robo3.1 is expressed before crossing, while Robo3.2 is expressed after crossing [54,72,73]. The authors carried out a series of electroporation both in the mouse and the chick embryo to search for isoform-specific functions. They observed in the chick embryo that Robo3.1 overexpression induces multiple midline recrossing. In contrast, Robo3.2 overexpression prevents the crossing. In the mouse embryo, Robo3 invalidation results in a failure of midline crossing $[72,73]$ and reexpression of Robo3.1 could restore the phenotype [72]. Thus Robo3.1 can be seen as a functional equivalent of Comm, preventing commissural axons which express Robo from responding to the midline Slits. The switch from Robo3.1 to Robo3.2 would activate the sensitivity once the crossing is accomplished.

The current questions relate to the nature of the mechanisms regulating the temporal and spatial distribution of the Robo3 isoforms along with the floor plate navigation as well as those regulating Robol/2 signaling, for example, 
are Robol/2 levels or cell surface expression regulated from precrossing to postcrossing stages and if so is this Robo3 dependent? Alternatively if the Robol/2 receptors are present at the cell surface from the onset unlike in the drosophila model, how are they maintained in an inactive state before crossing?

First, proteolytic processing of Robo receptors could account for such process. The metalloprotease of the Adam family Kuzbanian (kuz) was shown to cleave Robo in drosophila [74]. This cleavage was moreover functionally required since the defects induced by kuzbanian mutation were not rescued by a form escaping the processing [74]. In vertebrates, Robo processing could be activated upon crossing to mediate the Slit signaling.

Second, ubiquitination of Robol has been reported to contribute to axon sensitivity to Slits [75]. By screening a mouse brain cDNA library in yeast two-hybrid strategy, Yuasa-Kawada and collaborators isolated clones encoding ubiquitin-specific protease 33 (USP33, also known as von Hippel-Lindau protein-interacting deubiquitinating enzyme 1, VDU1). Using biochemical approaches, the authors showed that the Robol intracellular domain interacts with USP33 and that Robol can be ubiquitinated. In culture, knockdown of UPS33 prevented commissural neurons from responding to Slit collapsing effect. Finally, when UPS33 transcripts are silenced by electroporation of siRNA in the chick neural tube, commissural axons were observed to stall at the midline. Thus Robol ubiquitination could be one of the mechanisms switching on the Robo/Slit signaling at the midline.

Third, recent study by the Stoeckli lab brought significant answers, implicating RabGDI (Rab Guanine Nucleotide Dissociation Inhibitor1), a component of the vesicle fusion machinery, in the regulation of Robol levels [76]. The authors reported that interfering with RabGDI prevents the delivery of Robol to the growth cone surface by a subset of vesicles. In the chick model, downregulation of RabGDI by electroporation of siRNAs resulted in stalling of commissural axons in the floor plate, a phenotype which was similarly induced by loss of Slit1. Loss of Slit2 had lower impact on floor plate crossing but induced ipsilateral returning of axons, a behavior that was much less frequently observed after loss of Slit1. The authors observed in explant cultures that expressing RabGDI in precrossing commissural neurons normally insensitive to Slit repulsion was sufficient to trigger a response. The authors postulated that RabGDI could regulate the Slit receptor levels and turned to a cellular model to investigate this possibility. In transfected COS cells, the typical perinuclear staining of Robol is redistributed to the cell membrane when coexpressed with RabGDI. The authors propose an interestingly model whereby Robol would be confined in vesicles until crossing because of low levels of RabGDI. After crossing, RabGDI upregulation would then allow for the delivery of Robol to the membrane [76].

As for Comm, the question of how Robo3 itself is regulated is also central to our understanding of midline crossing. The literature reports differences depending on the CNS structures. In the spinal cord, Wilson and colleagues reported that Robo3 is a direct target of Lhx2 and Lhx9, those genes specifying the commissural feature of the projection patterns of Dll neurons [25]. First, the authors observed that in the Lhx mutants, commissural axons lack Robo3 expression. Second, they found in the Robo3 sequence consensus sites for Lhx2 and Lhx9. Finally, using EMSA (Electrophoretic Mobility Shift Assay) they showed that Robo3 expression is under the direct control of Lhx2 and Lhx9 in dll commissural neurons. This transcriptional pathway is not general as Robo3 is expressed in precerebellar neurons lacking Lhx2 and Lhx9. The nature of the genetic program controlling Robo3 in this system is unknown, but Robo3 levels were found regulated by the RNA binding protein Musashil [77]. Interestingly, the authors observed in explant assays that the Msil activity is suppressed by local floor plate signals. Thus, this raises the intriguing possibility that Robo3 levels are locally regulated when commissural growth cones navigate the floor plate.

A yet poorly known and important aspect of the midline crossing process is the spatial distribution of the gradients of repellents. The formation of morphogen gradients, whose effects on cell fates are concentrationdependent, has been particularly investigated in drosophila in the past years. Experimental and theoretical models envisage in addition to passive diffusion interactions with cell surface receptors that restrict the spreading of the diffusible cue and shape the gradient [78]. Extracellular matrix molecules, particularly the heparan sulfate proteoglycans (HSPGs), were identified as important binding components for morphogens [79]. Several years ago, Hu reported that Slit2-Robo-1 interactions are enhanced by HSPGs [80]. The HSPG Syndecan was also shown to contribute to the spatial distribution of Slits at the midline of the Drosophila ventral cord [81]. More recently, solid-phase ELISA binding assays were conducted which revealed an interaction between Slitl and Collagen IV, an ECM component of the basement membrane. This study showed that Collagen IV establishes a scaffold at the surface of the tectum, which contains slits, and is critical for the laminar-specific targeting of retinal ganglion cell axons [82]. Wright and collaborators provided now evidence for a role of dystroglycan in localizing Slits in the ventral spinal cord [83]. As is the case for other dystroglycan interacting components, Slits contain a dystroglycan-binding Laminin G (LG) domain in its C-ter region. The authors observed that many commissural axons are stalled in the floor plate and failed to cross it in mice lacking dystroglycan, which distributes to the basement membrane surrounding the spinal cord and in the floor plate. Similar defects were found in mouse lines carrying loss of function mutations in enzymes of the dystroglycan glycosylation machinery. To show that dystroglycan localizes Slit proteins in vivo, the authors performed binding experiments using Robo-AP probes. The staining pattern observed in the floor plate and in the basement membrane of the wild types was abrogated in mutants with defective dystroglycan glycosylation. This study raises several issues. First, Slits were found to be processed into a N-ter fragment carrying the LRR2 Robo binding domain essential for Slit chemorepulsive activity, and a C-ter fragment with yet unknown function, shown here to possess the dystroglycan binding domain. Two heparin binding sites have been mapped in each fragment, with the N-ter heparin binding stabilizing the Robo-Slit interaction 
and the other motif having yet unreported activity. The Slit proteolytic cleavage is nevertheless not indispensable for Slit activity in Drosophila since reexpression of a Slit form escaping the processing in a null background could restore the commissures [74]. In vertebrates, the functional significance of the Slit processing is yet unknown. Depending on this issue, the results can be interpreted in different ways. Since dystroglycan loss of function affects commissural axon guidance, one first possibility would be that it localizes the full-length (active) protein. Alternatively, it would localize the C-ter fragment this implies then that this fragment has a bioactivity on commissural axons.

(2) The Ephrin-Eph Signaling. The Eph-Ephrin signaling has been largely implicated in the navigation of commissural axons across the midline in various systems, particularly the corticospinal tract decussating at the spinal cord entry [84, 85] and the optic tract at the chiasm [86]. Interestingly, EphB2 mediating EphrinB3 repulsion was shown as a downstream target of the zic2-dependent transcriptional program specifying the ipsilateral pattern of retinal ganglion cell neurons [86]. The contribution of the Ephrin signaling in the navigation of spinal commissural axon at the floor plate has not been deeply investigated yet. Nevertheless, EphB receptors were found expressed by spinal commissural neurons and EphrinB3 by floor plate cells. Kadison and collaborators reported that mutant mice for EphrinB3 and EphB receptors exhibit a variety of guidance errors at the midline, for example, zigzagging trajectories during floor plate crossing, highly suggestive of weakened repulsive forces [87].

(3) The Semaphorin Signaling. In addition to Slits, Semaphorins act in the floor plate and regulate midline crossing. The class 3 Semaphorins (Sema3s) are secreted molecules in vertebrates that activate receptor complexes composed of Neuropilin (Nrp, 1 or 2) and signaling co-receptor of the Plexin-A (PlexA) family [88]. Additional Neuropilin coreceptors have been shown to be required for the growth cone responsiveness to Sema3s, such as the Ig Superfamily Cell Adhesion Molecules L1-CAMs [89]. In the spinal cord, Sema3B mRNA is specifically detected in the floor plate. Sema3B is a selective repellent for postcrossing commissural axons in explant cultures [71]. Nawabi and collaborators [51] showed that mice lacking Sema3B or its signaling coreceptor PlexinA1 present similar commissural defects of stalling and premature turning in the floor plate. These defects are also present in mice lacking the ligand binding subunit of the receptor, Nrp2 [71]. The Castellani lab characterized a regulatory pathway by which the sensitivity of commissural axons to Sema3B is silenced before crossing and switched on after crossing [51]. The authors showed that although PlexinA1 and Nrp2 are synthesized by commissural neurons, an endogenous protease found to be Calpainl, processing PlexinA1 to prevent its expression at the growth cone surface. The authors fused PlexinAl to the GFP Ph sensitive PhLuo, which fluoresces at neutral $\mathrm{Ph}$, to visualize the cell surface protein pool. The vector was electroporated at low levels in the chick neural tube and the distribution of fluorescent growth cones before and after crossing quantified. The vast majority of fluorescent growth cones were present in the postcrossing compartment, unlike control growth cones expressing the classical GFP that were present in both compartments. This active mechanism of PlexinA1 processing results in the silencing of the responsiveness to Sema3B until commissural growth cones cross the midline. How is the Sema3B responsiveness switched on? The authors produced floor plate conditioned medium, applied it to commissural cultures, and observed that calpainl activity and PlexinAl processing were suppressed by cues present in the conditioned medium. The treatment resulted in a gain of sensitivity to Sema3B. Thus, an extracellular signaling active in the floor plate controls the sensitization of commissural growth cone to the midline sema3B repellent $[50,51]$.

Recent investigation of the nature of the active floor plate components led the Castellani lab to identify a dual signaling mediated by the Ig Superfamily Cell Adhesion Molecule and the Neurotrophic factor gdnf [52]. Restricted sources of NrCAM and gdnf were detected in the floor plate. Gdnf deletion in mice resulted in commissural defects of floor plate crossing, which were aggravated when $\mathrm{NrCAM}$ was invalidated. In vitro gdnf and NrCAM both regulated calpain1 activity, PlexinA1 levels and switched on the sensitivity of commissural growth cones to Sema3B. Moreover, Floor plate conditioned medium produced form mice lacking NrCAM or gdnf displayed limited functional properties, compared to controls. Two different receptors are known to mediate the gdnf effects, the tyrosine kinase RET, and the cell adhesion molecule NCAM. NCAM was the only one found expressed by commissural axons, and commissural neurons isolated from the NCAM mutant were unable to respond to the gdnf effect. In addition and as expected NCAM invalidation in mice mimicked the floor plate crossing defects of gdnf deletion (as well as those of Sema3B and PlexinA1) [52].

Another study reported the implication of Shh in switching on the sensitivity to Sema3B and to another Sema3, Sema3F, which lacks at the floor plate but is expressed in lateral ventral domains. The authors reported that inhibiting Shh activity in spinal cord open books with a functionblocking antibody or with an inhibitor of the Shh receptor Patched-1 induced floor plate crossing defects which resemble those resulting from invalidation of the Semaphorin signaling [90]. They proposed that Shh application to commissural neurons decreases PKA activity, turning on their sensitivity to Sema3s. How these different floor plate signals are integrated in commissural growth cones is still unclear.

2.3.2. Switching off the Floor Plate Attractants. An important concept of commissural axon navigation in the floor plate emerged from early finding that commissural axons loose their responsiveness to Netrin1 after crossing [56]. In clever experiments, Shirasaki and collaborators cultured spinal cord open books from rat embryos and grafted floor plate tissue at lateral position, either at precrossing position (before the axons reached the endogenous floor plate) or at postcrossing position. They observed that precrossing commissural axons were rerouted towards the ectopic floor plate, whereas postcrossing axons were not. In xenopus spinal neurons, Netrin attraction was shown silenced in the presence of 
Slit, which induced formation of a complex between Robo and DCC receptors in commissural axons that resulted in inhibition of DCC signaling activity [91]. These data bridging floor plate attractants and repellents built the concept that navigation at the floor plate relies on a stepwise program orchestrated by series of regulation of growth cone responsiveness modifying the perception of the local environment to allow its entry, its crossing, and its exit of the floor plate.

Although it might efficiently convert an initial target into a nonpermissive territory, loss of sensitivity to attractants together with gain of sensitivity to repellents appeared surprisingly not entirely sufficient to drive commissural axons out of the floor plate after midline crossing. At least two additional mechanisms are required, one to stimulate axon growth, the other to switch an initially attractive signal, Shh, into a repulsive one. Gore and colleagues found that the Stem Cell Factor (SCF) expressed in the floor plate specifically acts on commissural axons expressing the Kit receptor after crossing to stimulate their growth [92]. In mice lacking SCF or Kit commissural axons stalled at the floor plate exit. A switch of Shh from attraction to repulsion upon crossing was suspected because the Charron lab and the Stoeckli lab independently reported that $\mathrm{Shh}$ has a precrossing attractive function in the mouse and a postcrossing repulsive action in the chicken. Additional investigations then established that this dual function is mediated by distinct $\mathrm{Sh}$ receptors, BOC at precrossing stage and HIP at postcrossing stage [44, 93]. This raises the intriguing question of how the transition from one receptor to another one is achieved. Recent study by Yam and collaborators brings insights into this question $[44,94]$. The authors observed in cultures of dorsal spinal cord neurons isolated from mouse embryos in Dunn chambers that commissural axons exposed to Shh gradient switched their response from attraction to repulsion after 3 to 4 days in culture, suggesting a cell-intrinsic and timedependent underlying mechanism. Such changing of growth cone responsiveness to guidance cues over time had also been observed in the Holt lab in the Xenopus visual system [95]. Initially insensitive when put in culture, retinal axons were shown to acquire responsiveness to Sema3A over $28 \mathrm{~h}$ to 32 hours of culture. In this system, the timer was identified as being the microRNA mir-124, regulating the transcript of COREST, which acts as a transcriptional repressor of the gene encoding the Sema3A receptor Neuropilin1 [95]. A distinct type of mechanism appears active in the switch of spinal commissural neuron response to Shh. Yam and collaborators identified a key contribution of 14-3-3 proteins. In vivo, 14$3-3 \beta$ and $14-3-3 \gamma$ are enriched in crossing and postcrossing segments, and in vitro their expression increases over time. Commissural neurons treated with an antagonistic peptide to inhibit 14-3-3 protein interactions failed to turn their attractive behavior to repulsion after 3 days in culture. Knockdown of 14-3-3 $\beta$ and 14-3-3 $\gamma$ siRNAs transduced in commissural neurons had similar outcome. Conversely, increasing 14-3-3 protein levels switched commissural axon attraction to repulsion. The authors also evidenced that interfering with 14-3-3 proteins in rat and chick embryos resulted in aberrant rostrocaudal choices, typically observed in context of shh loss of repulsive function. Additional investigations of downstream signaling suggest that 14-3-3 proteins might act via decrease of PKA activity [94].

2.3.3. Regulating Cell Adhesion. Stoeckli and Landmesser were the first investigators to demonstrate the crucial importance of cell adhesion molecules during commissural axon navigation. Their early work established that interfering with Ig Superfamily Cell Adhesion Molecules expressed by commissural axons and floor plate cells by injection of function blocking antibodies or recombinant molecules impairs floor plate crossing and exit $[96,97]$. Burstyn and collaborators investigated the function of F-Spondin, a secreted cell adhesion molecule observed to be highly expressed by floor plate cells. Injection of F-Spondin in the lumen of the central canal resulted in premature longitudinal turning [98]. In the mouse, L1-CAM distributes specifically in the crossing and postcrossing segments of commissural axons. When L1CAM expression is prematurely forced so that the molecule is already present in precrossing axons, it induces stalling of commissural axons in the floor plate [99]. Thus over their navigation, commissural axons engage complex and dynamic cell adhesion forces with their environment, which need to be coordinated with the guidance decisions taken by the growth cones. Very little is yet known on how these adherent and guidance forces are integrated.

\subsection{Navigation of Commissural Axons: Step 3: Postcrossing Guidance Decisions}

2.4.1. Specification of the Rostro-Caudal Binary Choice. Commissural axons leaving the floor plate must make new guidance decisions in order to turn and navigate longitudinally to the floor plate. Dl1 and dl2 commissural neurons make distinct choices, dllc neurons project their axons rostrally after crossing, while $\mathrm{dl} 2$ neurons project both rostrally and caudally.

Avraham and collaborators investigated the role of the Lhx transcription factors in the specification of this rostrocaudal binary choice, with genetic assays in the chick model [100]. Using in ovo electroporation, the authors tested and characterized new enhancing elements driving the gfp in dl1 and $\mathrm{dl} 2$ neurons, thus allowing the specific monitoring of their axon trajectories. They then manipulated the lhx code in dl neurons and investigated whether it impacted on their rostrocaudal choice. This interesting study brought the conclusion that Lhx9 and Lhxl control the rostrocaudal choice, with Lhxl triggering rostral turning and Lxh9 triggering the caudal one.

(1) Action of Chemotropic Gradients. At protein level, the rostrocaudal orientation of the commissural postcrossing navigation was shown to depend on gradients of secreted molecules. At least two opposite gradients act synergistically, one is a caudal-to-rostral Shh gradient having repulsive activity, the other one is an opposite rostral-to-caudal Wnt attractive gradient $[93,101]$. Several Wnts, including Wnt4, Wnt5a, Wnt7a, and Wnt7b were found to establish high rostral to low caudal gradients in the neural tube, which attract 
commissural axons [101]. Conversely in the chick embryo, Shh forms a high caudal to low rostral gradient, which repels commissural axons [93]. These two gradients might act in synergy to shape the rostrocaudal turning of postcrossing commissural axons. More recently, Domanitskaya and collaborators examined whether the two anteroposterior Shh and Wnt signaling could act cooperatively [102]. First, the authors searched for Wnt candidates that would have an appropriate profile to set an anteroposterior gradient in the chick embryo, as implication of the Wnt signaling had been reported in the mouse embryo. They concentrated on Wnt5a and Wnt7, expressed in the floor plate and adjacent tissue. Loss of function experiments, however, suggested that the anteroposterior guidance activity of the Wnts was conserved in the chick. Nevertheless and unlike Wnt4 in the mouse embryo, Wnts were not found to form an anteroposterior gradient in the chick spinal cord. The authors uncovered a mechanism explaining the apparent discrepancy between Wnt expression and function. They found that the Wnt antagonist Sfrp1 (for Secreted Frizzled-related protein1), known to be regulated by Shh, had in contrast a graded expression, thus generating an anteroposterior gradient of Wnt activity. Thus, by regulating sfrp1, Shh gradient could shape an opposite graded Wnt activity along the anteroposterior axis, thus reinforcing the topographic information for postcrossing axons [102].

(2) Action of Cell Adhesion Molecules. Cell adhesion molecules also play prominent roles during postcrossing commissural axon navigation. Once commissural growth cones have accomplished their rostrocaudal choice, they navigate longitudinally towards their target cells. This step of the navigation was shown by the Stoeckli lab to require contribution of cell adhesion molecules. Likewise, evidence from investigations in the chick model revealed that the recently discovered Ig Superfamily cell Adhesion Molecule MDGA2 is expressed by spinal commissural neurons [103]. In ovo knockdown of MDGA2 resulted in lack of longitudinal growth. In their study, the authors propose that through MDGA2 homophilic binding, postcrossing commissural axons and ipsilateral axons could fasciculate for navigating together. The Necl/SynCAM subgroup of Ig Superfamily Cell Adhesion Molecules, initially discovered for its role during synaptogenesis, is also active during commissural axon guidance. $\mathrm{Nec} /$ Syncam was found expressed by commissural axons and floor plate cells. Knockdown by RNAi strategy in the chick embryo resulted in aberrant behaviours at the floor plate exit, thus demonstrating that these molecules are needed for the postcrossing step of commissural axon navigation. Targeted loss-of-function in floor plate cells or in commissural neurons demonstrated that both sources are required for proper navigation at the floor plate. Based on their analysis of interaction profiles, the authors propose that Necl/SynCAMs act via heterophilic trans interactions between $\mathrm{Necl} 2$ and Necl3 family members [104]. How these distinct cell adhesion molecules coordinate their action to accomplish the transition between floor plate exit and longitudinal turning is one of the questions that should be investigated in the future.
2.4.2. Organization of Longitudinal Commissural Tracts. (1) Robo Code for Longitudinal Axons. The organization of postcrossing longitudinal axon tracts has been particularly explored in the drosophila ventral cord. Post-crossing commissural axons are arranged in three distinct tracts coursing parallel to the midline: Robo 2 and Robo3 but not Robo1 were shown to control this sorting $[105,106]$. To decipher the features distinguishing Robo proteins, Spitzweck and collaborators $[67,107]$ generated transgenic flies in which the coding sequence of one Robo was introduced into the loci of another one "Robo swaps". The authors observed that rather than functional differences, it is the Robo expression profile on postcrossing axons that accounts for their lateral sorting. Thus, exciting insights should come from investigation of the mechanisms controlling the Robo distribution after midline crossing.

In vertebrates, postcrossing commissural axons also select distinct mediolateral positions, although individualized tracts are not as distinct as in the fly. Specific Robol and Robo 2 requirement for postcrossing commissural navigation in Vertebrates has been shown by the Tessier-Lavigne lab who compared the phenotypes of mouse embryos lacking Robol and Robo2. Loss of Robol reduced the thickness of the ventral funiculus, while loss of Robo2 impacted on the thickness of the lateral funiculus [108]. Thus functional specificities distinguishing Robol and Robo2 could exist which sets the lateral position of post-crossing commissural tracts. Alternatively, distinct expression profiles and/or levels of Robol and Robo 2 are responsible for the lateral arrangement of the tracts. In their study Jaworski and collaborators also described an additional role for Robos, which is to restrict postcrossing commissural axons out of the grey matter. The authors propose that this function might be mediated by a second source of Slits, the motoneurons [108].

(2) Selective Fasciculation and Longitudinal Axons. The genetic markers of $\mathrm{dl} 1$ and $\mathrm{dl} 2$ neurons in the chick embryo developed by Avraham and collaborators allowed them to visualize cell-type specific tracts over all others having similar trajectories [100]. This gave the authors the opportunity to observe that dll and $\mathrm{dl} 2$ axons self-fasciculate forming distinct tracts, the dll tract lying ventrally to the $\mathrm{dl} 2$ tract. Moreover, the authors also found that the dlic arriving in the contralateral side fasciculated onto the dlli axons, forming common cell-type specific bundle. This provides the possibility for the ipsilateral tracts to contribute to the orientation of their homologous commissural tracts after crossing. Up to now, investigations of mouse models defective for spinal cord floor plate cues have focused on commissural (and not ipsilateral) axon trajectories. Lack of genetic commissural and ipsilateral markers in these models has hampered further investigation of this possibility. The selective fasciculation is generally mediated by cell adhesion molecules. It is notable that several guidance receptors, among which are the Semaphorin receptors Nrps and Plexins, have been originally identified for their axon fasciculation properties [109]. It is plausible that in addition to their role in axon guidance choices, these receptors also mediate some axonaxon recognition. Moreover, the distribution of "classical" 
cell adhesion molecules in commissural axons is strikingly dynamic and in some cases varies from the precrossing to the postcrossing stages. Likewise, in rodents L1-CAM is distributed in the crossing and postcrossing axon segments, unlike Tag-1, another IgSFCAM, which exhibits the strict complementary profile, is only present in the precrossing axon segments. In the chick, these cell adhesion molecules are distributed differently but are also compartmentalized along the axon shaft $[99,110,111]$. Thus it is still difficult to evaluate the contribution of fasciculation and axon-axon recognition processes in the guidance of postcrossing commissural tracts. Investigations will certainly greatly benefit from the generation of transgenic mouse lines with cell-type specific fluorescent axon reporters.

\section{Navigation of Axons Forming the Corpus Callosum}

3.1. Specification of Callosal Cortical Neurons. The callosal projections interconnect precise populations of cortical neurons and are integrated in a circuitry dedicated to the processing of highly complex brain functions [2]. The cerebral cortex is composed of hundreds of different neuron types and is subdivided into functionally and morphologically distinct areas and layers, each of them possessing their own pattern of input and output. Several cortical layers participate in callosal projections, the superficial layers $2 / 3$ for the vast majority of them and deep layer 5 in a minor proportion. The molecular program orchestrating the specification of the different cortical subtypes is the subject of extensive investigations [2]. Several transcription factors and regulators have been characterized as part of the program assigning callosal and subcortical identity to cortical pyramidal neurons. Likewise, the zinc finger transcription factors Scip2 and Fezl2 participate in the program specifying cortical neurons with subcortical projections [112]. Alcamo and collaborators found that the DNA-binding protein Satb2 is expressed in cortical neurons of layers 2 to 5 that project an axon across the corpus callosum. In mice deficient for Satb2, these axons were found rerouted in subcortical pathways, following axons of the corticospinal tract [113]. Thus Satb2 is a major determinant of the callosal identity. In a more recent study, the transcriptional regulator ski was shown to form a complex with Satb2, being part of the chromatin remodeling complex binding to regulatory sequence of Ctip2. By recruiting the transcriptional repressor to the Ctip2 locus, it suppresses the genetic program specifying subcortical projections, thus promoting the callosal identity [114]. Overall and as described for the contralateral/ipsilateral subdivision in the spinal circuits, the callosal/subcortical projection choice is controlled by a genetic program.

3.2. Callosal Navigation, Step 1: Medial Turning Decision. Callosal projections radiate from the cortical surface, interconnecting homologous left and right cortical areas. The navigation of callosal axons can be subdivided into a series of steps, the initial radial navigation towards the white matter side, the medio-lateral turning at the cortex exit, the tangen- tial course across the midline, and the final radial ingrowth in the cortex at the corresponding contralateral side. Hand and Polleux reported that the medial turning typical of callosal axons and distinguishing them from the rest of cortical neurons projecting to subcortical structures that turn laterally is controlled by the transcription factor Neurogenin2 (Ngn2) [115]. In mice lacking Ngn2, the corpus callosum was found reduced by $50 \%$. The authors conducted an investigation of the early stages of callosal navigation. They knockeddown Ngn2 with shRNA by in utero electroporation of the dorsal telencephalon and prepared organotypic slice cultures to assess callosal axon behaviors. Interestingly, knockdown of Ngn2 resulted in abnormal lateral instead of medial turning, revealing errors in the early step of callosal navigation. Several genes encoding guidance receptors have been found as direct targets of Ngn2 [116], thus suggesting that Ngn2 sets a specific guidance receptor code for callosal axons which allows them to make the binary lateral-medial choice appropriate of their identity. The nature of the guidance signaling underlying this medio-lateral choice remains to be determined.

\subsection{Navigation across the Midline}

3.3.1. Role of Guidepost Cells in the Formation of the Corpus Callosum. (1) The Midline Glia. In contrast to spinal commissural axons that cross the midline ventrally, callosal axons navigate across the midline dorsally and as a consequence need the two hemispheres to be fused, to provide them with a bridge. Indeed, many failures of midline crossing directly result from improper fusion of the two hemispheres. For example, in mice, mutation of chd7, a chromosomehelicase-DNA-binding protein, causes alterations of the dorsal midline in the telencephalon which expands and is not properly invaginated, then preventing corpus callosum formation. Interestingly this mouse model recapitulates the neuro-developmental disorders of patients suffering from the CHARGE syndrome. Chd7 was found to bind an enhancer downstream of BMP4. In the mouse model, BMP levels and spatial expression pattern were found to be affected at the midline, thus being likely responsible for the dysgenesis of the dorsal midline [117]. The spinal cord and brain midline environment share a contribution from the glial lineage. Thus, the cells that fulfill the bridging function at the dorsal midline of the brain are of glial origin. They arise from radial glial cells lying in the ventricular zone of the cerebral cortex. At the dorsal midline, these cells differentiate into astrocytes much earlier than in other parts of the cerebral cortex and start expressing the GFAP marker well before birth [118]. In addition and as described in the next paragraphs, the midline glia provides guidance cues to callosal axons which channel their navigation and prevent them from entering adjacent inappropriate territories. Thus, the midline glia plays an instructive role in the guidance of callosal axons, in a manner highly similar to the floor plate cells for spinal commissural axons (Figure 4(a)).

Twenty years ago, three distinct glial populations were discovered to populate the midline: the glial wedge, the indusium griseum glia, and the midline glia zipper [118]. 


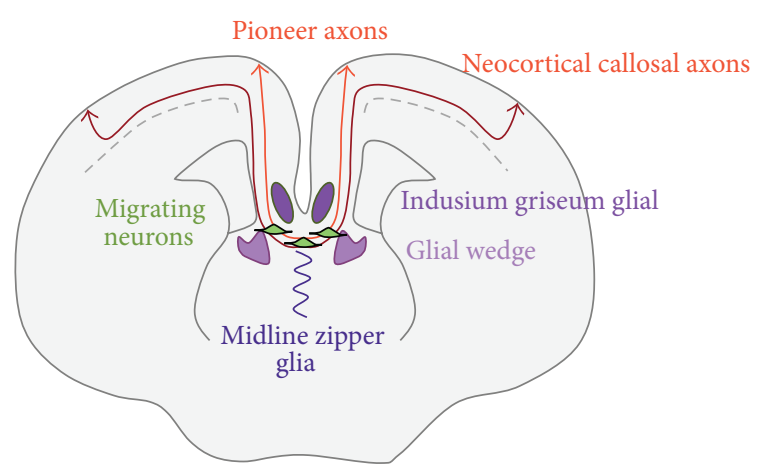

(a)

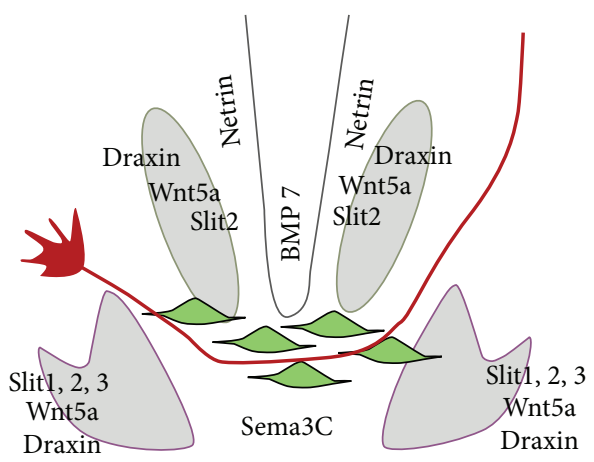

(b)

FIGURE 4: Guideposts and guidance signaling controlling the corpus callosum formation. (a) Axons from the cingulate cortex (in orange) pioneer the corpus callosum. Neocortical callosal axons (in red) turn medially and navigate towards the midline. The axons are guided by cues emanating from various sources, the midline glia, migrating neurons, and meninges. (b) Summary of the major guidance cues guiding the callosal axons across the midline.

The glia zipper is assumed to ensure the fusion of the telencephalic hemispheres. These glial populations contribute to the navigation of callosal axons across the midline by providing them with guidance cues. As a consequence, alteration of their distribution or differentiation impacts on the development of callosal axons. The Richards lab extensively studied the development and the function of midline glia cells [119]. Piper and collaborators, for example, showed that The Nuclear Factor One gene family of transcription factors plays a role in gliogenesis at the brain dorsal midline, as it does also in the spinal cord [120]. Mice deficient for Nfib exhibit callosal defects, although callosal neurons only express low levels of Nfib. This prompted the authors to investigate noncell autonomous contribution of Nfib, and they could provide evidence that lack of Nfib impairs the differentiation of radial progenitors into mature glia. Magnani and collaborators investigated the potential role of the zinc finger transcription factor gli3 during callosal development. Their analysis of mice with reduced gli3 levels together with transplantation experiments in slice cultures revealed that gli3 makes several contributions, among which the positioning of midline guideposts. In the gli3 hypomorphic mutant Pdn, ectopic glial cells are detected along the callosal path [121]. Nevertheless the wide expression of gli3 in the developing forebrain prevented further mechanistic interpretation of these defects. In a very recent work, the same lab used a conditional knockout approach to assess this issue. The authors could show that gli3 is required in cortical progenitors giving rise to callosal projection neurons and midline cells and not in adjacent territories for proper callosal navigation across the midline [122]. Further investigations of the neuronal and glial populations with specific markers support a prominent role of gli3 in the midline glia rather than in the projection neurons. In absence of gli3, neurons appeared normally specified, while in contrast glial wedge cells prematurely translocated to the pial surface forming a physical barrier for callosal axons [122].

The FGF pathway also has major contribution in midline glia positioning. In mutant mice specifically lacking FGFR1 in the telencephalon, callosal axons totally fail to cross the midline. Instead they form Probst-like bundles, a typical defect of corpus callosum formation [123]. In these mutants, all the three glial lineages (the indusium griseum glia, the midline glia zipper, and the glial wedge) are also missing. This made it difficult to further assess the cause of the defect and understanding whether FGFR1 is required in callosal neurons and/or in glial guideposts. Smith and collaborators generated a mouse line with targeted inactivation of FGFR1 in radial glia and observed that it totally disrupted the indusium griseum astroglia and also altered the glial wedge in anterior regions of the cerebral cortex [124]. This resulted in a lack of astrocyte accumulation at the midline. The authors studied the development of the midline astroglia and found that the radial glia detaches their apical anchor to translocate to the pia and migrates to build the indusium griseum. In absence of FGFR1, the cells are unable to properly achieve their translocation to the pia. Interestingly, the selective ablation of FGFR1 in neurons neither impacted on the corpus callosum formation nor on the development of the midline glia. Thus, the dysgenesis of the corpus callosum observed after FGFR1 removal in the astrocyte lineage can be imputable to defective midline glia.

Contribution of the TGF $\beta$ signaling to the differentiation of the midline glia was also reported in the recent literature [125]. Sánchez-Camacho and collaborators observed an expression of BMP7 in the presumptive tissue of the midline glia and found that the genetic loss of BMP7 in mice results in profound alterations of the glial wedge and the indusium griseum glia. The authors infused BMP7 protein in the developing forebrain and observed that it resulted in premature differentiation of the midline glia. As expected, these in vivo manipulations of BMP7 impaired the callosal navigation across the dorsal midline.

In 2012, the Richards lab reported that the Slit proteins contribute to the positioning of midline glia [126]. Analysis of the glial population in mice lacking Slit2 or Slit1 and Slit2 revealed that the indusium glia was ventrally shifted relative to the glial wedge. This likely explains part of the defects of callosal navigation observed in these mutant mice. Interestingly, upregulation of Slit 2 has been noted in the Pdn mutant, supporting a prominent role for the Slit signaling in the positioning of midline glia [121]. A recent study performed 
on forebrain commissure formation in the zebrafish model reported that Wnt-8b and Frizzled3a cooperate to control discrete Slit2 expression in the commissural plate. As reported in the mouse brain, Slit2 appeared essential for proper differentiation of the midline glial structures acting as guideposts for the forebrain commissures [127]. Thus, one of the first causes of corpus callosum agenesis and dysgenesis is the defective differentiation and/or migration of the midline glia.

Overall, a complex molecular cascade which includes key developmental pathways appears to control the differentiation of the glial lineages acting as guideposts for the corpus callosum formation.

(2) Transient Neuronal Guideposts. Additional populations of cells, of neuronal origin, have been recently discovered which also act as guideposts for callosal axons (Figure 4(a)). Alterations of the developmental programs controlling the spatial positioning of these neuronal guidepost cells were shown to result in defective midline crossing. The Lebrand lab explored the cellular organization of the embryonic forebrain dorsal midline and noticed that numerous GABAergic and glutamatergic (Calretinin $\mathrm{CR}^{+}$) neurons populate the developing corpus callosum and the glial sling [128]. The authors further mapped the precise localization of these cells in relation with the progression of callosal axons. They observed that prior to the arrival of callosal axons (between E12.5 and E15.5 in the mouse), the $\mathrm{CR}^{+}$neurons occupy a key position at the midline. By the time the first axons cross the midline, which is E16.5 in the mouse, and the GABAergic neurons mix with the $\mathrm{CR}^{+}$neurons at the midline. At E18.5 the glutamatergic neurons organize in three distinct stripes within the corpus callosum, delineating the dorsal and ventral borders of the tract. After birth, the glutamatergic lineage disappears rapidlys while the GABAergic lineage persists a few days longer. Analysis from mouse genetic approaches and ex vivo transplantation experiments allowed the authors to assign a guidepost function to these transient neuronal populations in corpus callosum formation. First, the CC was found to be severely impaired in mice lacking the GABAergic lineage. Transplantation experiments in slice cultures indicated that this is not due to unexpected defect of callosal projection neurons but rather to alterations of the corpus callosum cellular environment. Thus these neuronal populations represent a novel type of guideposts and their proper differentiation and positioning might be essential for the formation of the corpus callosum, as it is the case for the glial guideposts. A recent study reported that the GABAergic guideposts are indeed a mix of two distinct populations originating from the medial and the caudal ganglionic eminences [129].

Another recent work uncovered an original role of the transcription factor RFX3, a gene involved in ciliogenesis, in the development of the callosal guideposts [130]. Malformations of the corpus callosum have been frequently observed in patients suffering from ciliopathies, but the underlying physiopathological mechanism remained very unclear. Benadiba and collaborators observed that the glial guideposts are affected by RFX3 deletion, and astrocytelike cells appeared mispositioned [130]. Interestingly and as expected from a key role of guidepost cells, mice lacking RFX3 develop an acallosal phenotype, in the most severe cases. In cases with milder phenotypes, the corpus callosum is hypoplastic. Complex transplantation experiments in slice cultures were performed on by the authors, which revealed that $\mathrm{RFX}^{++} /$midline explants rescued callosal midline crossing in $\mathrm{RFX3}^{-/-}$slices. Conversely, $\mathrm{RFX}^{-/-}$midline tissue transplanted in $\mathrm{RFX}^{+/+}$host slices prevented callosal crossing. Thus, the defect of callosal midline crossing in the RFX3 mutant mice results from a defective midline environment [130]. Interestingly, RFX3 expression was observed to colocalize with markers of these neuronal guidepost cells. RFX3 deletion in mice impairs the normal distribution of neuronal guideposts. Instead of forming a band across the midline, cells accumulated at each border. Cells were also found scattered in adjacent tissue. Some neuronal subpopulations however, normally distributed in the RFX3 mutant context [130].

Neocortical callosal axons also use another type of neuronal support. In early work, Koester and O'Leary discovered that axons arising from the cingulate cortex develop first and pioneer to the callosal pathway, crossing the midline at E15 in the mouse [131] (Figure 4(a)).

(3) Meningeal Tissue. Very recently, another source of guidance information for callosal axons has been discovered, that is, the meningeal tissue at the vicinity of the corpus callosum. Several recent works established unexpected and important contribution of signals derived from the meninges to the development of the cerebral cortex [132, 133]. Choe and collaborators generated mouse models in which the meninges are either produced in excess or are reduced at late developmental stage to limit early deleterious consequences [134]. Interestingly, alterations of the meninges resulted in strong defects of corpus callosum formation. In the loss of function model, the corpus callosum was enlarged, with an increased number of axons crossing at all rostrocaudal levels. Conversely in the gain of function model, callosal axons failed to cross the midline and were arrested on each side. This was not due to alterations of the glia guideposts and the cortical projection neurons. In contrast, expansion of the meninges over the surface of the cortex and in the interhemispheric fissure just adjacent to the site where the corpus callosum should form was clear. The authors provided evidence that the meningeal tissue overgrowth observed in their mouse line results from elevation of Wnt6 emanating from the skin. In the normal situation, Wnt6 expression in the skin is laterally high, dorsally low, and absent at the midline. In their mouse model, high wnt6 expression expanded at dorsal level. Thus, this study provided evidence that meningeal tissue contributes to the formation of the corpus callosum. As discussed in the next section, meninges are an essential source of morphogen signaling, which controls the outgrowth of callosal axons.

3.3.2. Guidance Signaling by the Midline Guideposts. Numerous studies were conducted to show that glial and neuronal guideposts provide positional information to callosal axons 
and to identify the nature of the underlying signaling. Probably unsurprisingly, most of the well-known axon guidance families were found to contribute to the navigation of callosal axons across the midline (Figure 4(b)). Contributions of axon guidance molecules were essentially investigated through the analysis of the callosal navigation in the corresponding knockout mouse models. It is likely that many of these guidance cues emanate from the midline guidepost cells. Nevertheless the exact contribution of each type of guideposts and the nature of the signaling that mediates their specific effects are far from being clear. Answers would require the selective inactivation of the candidate signaling in a celltype specific manner, and more specific markers than those already available would be required.

\section{(1) Guidance Signaling Provided by the Midline Glia}

The Wnt/Frizzled Signaling. The midline glia was reported to express Wnt family members. Likewise, Wnt5a is detected in the indusium griseum and the glial wedge. Mice deleted for the Wnt receptor Frizzled3 (Fz3) exhibit defects of brain commissures, including the corpus callosum [135]. In the context of deletion of another Wnt receptor, Ryk, the callosal tract becomes defasciculated and axons do not exit the midline region [136]. This is suggestive of a repulsive effect of Wnt signaling, and in agreement, Wnt5a could repel cortical explants in coculture experiments [136].

The Robo/Slit Signaling. The Robo/Slit signaling was also shown to play several (and distinct) roles during callosal navigation $[126,137,138]$. Recently, the Richards lab conducted a thorough investigation of the expression profile of Slit, and Robo family members at the time callosal axons are navigating the dorsal midline [126]. Using the glial marker GFAP in immunohistochemistry on brain sections, the authors could establish that although Slit1, 2, 3, and Robol, 2 are all detected in the indusium griseum, Slit 2 is the only one to have a glial origin. Furthermore, its expression is graded along the rostrocaudal axis. In contrast, cells of the glial wedge express all the Slit and Robo family members. Thus the midline glial guideposts produce complex combinations of Slits along the path of callosal axons. Differential contribution of the Slits is reflected in the nature of the corpus callosum alterations observed in the knockout models of the Robo/Slit signaling. While Slit1 loss has no impact, Slit2 deletion results in alterations, with some (but not all) axons stalling at the midline or coursing in aberrant directions [126, 137, 138]. Moreover, the tract is found ventrally displaced in context of Slit2 deletion. Removal of one or two copies of Slit1 and/or Slit2 allowed Unni and collaborators to establish that Slit1 does indeed make a contribution, since its deletion aggravates the defects resulting from Slit2 loss, leading to total corpus callosum agenesis. Another type of defect was detected in the Slit3 mutants, with a partial penetrance, consisting of a loss of corpus callosum at rostral but not caudal levels. Similar analysis of loss of one or two Slit alleles was conducted on Slitl and Slit3 mouse lines, which revealed that these two Slits regulate distinct aspects of the callosal navigation. Overall, a hierarchy in the Slit requirement emerges with
Slit2 having the major role and the two other Slits making minor contributions. The contributions of Robol and Robo2 receptors were assessed through the analysis of the corresponding knockout mouse models [126]. Interestingly, loss of both Robo1 and Robo2 does not fully recapitulate the Slit2 deletion, indicating that another and yet unknown Slit2 receptor might be present in callosal axons to mediate some of the Slit2 functions. Nevertheless conclusions must be drawn in the light of additional observations. For example in the Slit 2 knockouts (and possibly not in the same way in the Robol/2 ones), the glial guideposts are mispositioned and thus are not fully competent for delivering the guidance cues to callosal axons at appropriate location.

The Draxin/DCC Signaling. The guidance of callosal axons gained a novel repellent, the Draxin, a secreted protein with high affinity for basement membrane, discovered in 2009 by Islam and collaborators [31]. In the Draxin mutant mice, callosal axons fail to cross the midline and course ventrally in the most severe cases. Colabeling of callosal axons with the glial marker GFAP led the authors to observe that the indusium griseum glia is absent in the strongly affected Draxin knockouts. Draxin is expressed by the midline glia, and in explant cultures, Draxin act as a repellent for cortical axons. This study establishes that Draxin is an additional glial-derived repellent for crossing callosal axons. The most severe phenotype associated with the loss of indusium griseum might be caused by the loss of additional repellents normally delivered by this glial population [31]. Draxin has a wide expression and its loss affects other brain commissures, as well as the spinal commissures as reported in previous part of the review. In 2011, the Tanaka lab investigated the mechanism of action of Draxin to identify the receptors mediating the Draxin effects, and surprisingly, Draxin was found to bind to Netrin receptors [139]. DCC in particular binds Draxin with high affinity and is required for Draxinmediated axon collapse of cortical explants. Finally, mice lacking one copy of DCC and one copy of Draxin have stronger defects of corpus callosum formation than mice lacking one copy of a single gene, thus providing evidence for interactions between Draxin and DCC genes.

The Semaphorin/Neuropilin Signaling. A contribution of this signaling to the navigation of callosal axons has been reported in several studies, although to date, there is no experimental evidence that it is mediated by the glial guideposts. Defects of corpus callosum formation were reported in mice expressing a form of Neuropilin1 incapable of binding the Semaphorin ligands [140]. In contrast, loss of Neuropilin2 (Nrp2) alters some brain commissures but not the corpus callosum [141, 142]. In 2006, Piper and collaborators observed that Nrp1 is expressed by pioneer axons of the cingulate cortex. The other receptor components of the Semaphorin signaling, the L1$\mathrm{CAM}$ and the Plexins, were also expressed by the pioneer axons [143]. Interestingly, pioneer axons were shown to be misguided when the Nrp1/Semaphorin binding is genetically abolished in mice, pointing to an early requirement of the Nrp1/Semaphorin signaling in the development of the corpus callosum. Interestingly and as introduced earlier in 
the review, mutations in the $\mathrm{X}$-linked L1-CAM gene in humans cause a severe neurological disorder, which includes abnormal development of the corpus callosum. L1-CAM loss of function in mice recapitulates this defect [144]. It should be noted that L1-CAM has multiple binding partners, among which are several other cell adhesion molecules of the IG Superfamily. It is yet unknown whether the alteration of the corpus callosum formation relies or not on the function of L1-CAM in the Sema3A receptor complex [144].

The Semaphorin sources exerting a guidance activity on the navigating callosal axons are also not clearly identified. Several Semaphorins were detected in territories adjacent to the dorsal midline [143], but their exact contribution to the callosal navigation remains unclear. No obvious defects were observed in mice deficient for Sema3A [143]. In contrast and as detailed later, Sema3C is the first reported guidance cue delivered by neuronal guideposts to attract navigating callosal axons [128]. This Semaphorin family member had already been shown to act as an attractant for pioneer axons of the cingulate cortex and found to be expressed in the indusium griseum glia [143]. Thus overall, Sema3C is certainly a crucial cue and is likely regulating several aspects of the navigation of callosal axons.

The Netrin/DCC Signaling. Early work by the Richards lab concentrated on the Netrin/DCC signaling and provided evidence for a major contribution to the formation of the corpus callosum [145]. In mice lacking Netrinl or DCC, callosal axons failed to cross the midline. They do not stall but rather form Probst bundles on each side of the midline or enter the adjacent septum. This resulted in severe corpus callosum agenesis. Callosal axons express DCC throughout their development, suggesting it that might be the receptor mediating the Netrinl effect. Until recently, very little was known on the mechanism of action of Netrinl. Indeed, Netrinl is not expressed by the midline glial cells and thus cannot act as a midline attractant for callosal axons as it does for spinal commissural axons. Nevertheless, recent work indicates that it is expressed by cortical territory adjacent to the midline Fothergill, and collaborators investigated the responsiveness of cortical axons to Netrinl and found that it varies over time. Indeed, Netrinl was able to attract callosal axons from the cingulate cortex, those axons that pioneer the corpus callosum but did not attract the axons extending from neocortical explants that constitute the core of the callosal tract [146]. Differences in DCC expression levels might explain the distinct responsiveness of the pioneers and their followers. Silencing of the callosal responsiveness to Netrin through inactivation of the receptor or its downstream signaling is an alternative explanation. Whatever the case, the lack of attractive function of Netrinl for callosal axons constitutes a notable difference between midline crossing in the brain and in the spinal cord.

Integration of Axon Guidance Signaling. The data discussed previous indicate that callosal axons express DCC but are not attracted by Netrin. DCC might thus be dedicated to the repulsive Draxin signaling that is acting at the midline. Alternatively, DCC could also mediate Netrin function for callosal axons other than attraction. Recent work supports this latter hypothesis. How callosal axons exposed to Slit2 cross the midline in the presence of repulsive Slit2 emanating from the glial wedge and the indusium griseum is a puzzling issue. Fothergill and collaborators could show that in addition to its attractive effect on the pioneers, Netrinl decreases the sensitivity of callosal axons to the Slit2 repellent, via their corresponding receptors DCC and Robol [146]. The authors propose that this modulatory effect of Netrin would allow callosal axons to navigate the midline. Then DCC would be downregulated, and the attenuating effect of Netrin on Slit2 repulsion would become inactive, thus allowing the callosal axons to leave the midline. It remains unclear how such modulation integrates the repulsive Draxin signaling also acting at the midline and mediated by DCC. Additional regulatory mechanisms remain to be discovered to understand why the callosal axons expressing DCC are not responsive to Netrin attraction.

(2) Guidance Signaling by the Neuronal Guideposts. Niquille and collaborators investigated the nature of the guidance activity exerted by the neuronal guideposts using transplantation approaches. They could prove the existence of an attractive activity emanating from both types of neuronal guideposts [128]. Using candidate approach, they identified the Semaphorin family member Sema3c as a first chemoattractant. Sema3C is detected in $\mathrm{CR}^{+}$neurons and has attractive properties on callosal axons which are exerted via the neuropilin1 receptor. Thus, the Sema3C cue would be delivered by the neurons transiently populating the corpus callosum to keep callosal axons along their pathway. Such interplay between migrating neuronal and axonal populations has been nicely evidenced in other systems [147], and altogether these different studies draw a fascinating picture of the coordinated orchestration of migratory pathways in the developing nervous system. Future studies should lead to the characterization of additional chemoattractants released from these newly discovered neuronal guideposts. Importantly, this study establishes the first contribution of attractants in the navigation of callosal axons at the midline.

(3) Guidance Signaling by the Meninges. In their investigation of the implication of meninges to callosal pathfinding, Choe and collaborators observed that the corpus callosum defects observed in excess of meninges were present in the absence of any particular alterations of the guidance signaling known to be required for midline crossing, such as the Slit, Semaphorin, Draxin, Wnt5a, and Ephrin signaling [134]. The authors hypothesized that the overgrowth meningeal tissue may result in excess of BMP signaling (known to be derived from the meninges), then impact on the callosal navigation. In agreement, BMP7 was found to be elevated in the meningeal tissue of the gain of meninges line, and this resulted in increased phosphorylation of BMP target in the adjacent cerebral cortex. The authors then overexpressed BMP7 by in utero electroporation and demonstrated that it disorganized the pioneer projections arising from the cingulate cortex and inhibited the corpus callosum formation. This is consistent with previous work reporting that mice lacking 
BMP7 are acallosal, although in this study this phenotype was attributed to midline glia defects [125]. Intriguingly, affecting the cingulate cortex in one side interfered with the growth of callosal tracts from both sides, suggesting that the colonization of the corpus callosum path needs communications between left and right cingulate axons. Because BMP7 is expressed in the midline meninges in the normal situation, its inhibitory action might be counterbalanced by another molecule also present at the cortical midline. Through expression screening, in vitro explant cultures, and in vivo rescue, the authors demonstrated that the antagonist of BMP7 is Wnt3. Thus in coculture experiments, midline cortical explants extended axons which failed to grow across COS cell aggregates secreting BMP7, while they did so when the cells were transduced to coexpress BMP7 and Wnt3. Wnt3 expression in mouse embryos with excess of meninges (and of BMP7 signaling) could restore a corpus callosum. The authors propose that a dual BMP7/wnt3 signaling sets the timing of the callosal navigation across the midline. Meningeal-derived BMP7 would inhibit the navigation of the pioneer axons across the midline until Wnt 3 is released from the cortical midline to release the inhibition and allow the crossing [134].

\section{General Conclusion and Future Directions}

Axon navigation across the midline in the brain and in the spinal cord has been investigated under drastically different angles. On the one hand in the spinal cord, much effort has been made in understanding how commissural axons can initially be attracted towards the midline and how they are expelled away after crossing. In contrast, this issue remains elusive in the context of callosal navigation. While contribution of repellents produced at the cortical midline has been documented for several years, the action of midline attractants has been largely ignored until recently.

The question of whether callosal axons vary their sensitivity to midline attractants and repellents, as spinal commissural axons do, to achieve the transition between precrossing and postcrossing, navigation remains obscure, although recent study suggests that it might be the case [146]. The findings that cross-regulations between attraction and repulsion might also take place during callosal navigation raise now the question of the underlying mechanisms. Some of them might be similar to those acting at the spinal cord midline. For example, the lack of neocortical callosal sensitivity to Netrinl could be achieved through cross-regulation with the Slit signaling. Investigating the expression levels of guidance receptors at transcriptional and posttranscriptional levels, as well as the formation of receptor macrocomplexes, in link with midline crossing is certainly an issue that should deserve interest.

On the other hand in the cerebral cortex, midline guideposts have been the focus of many studies. This led to unexpected findings, such as the contribution of transient neuronal populations to the guidance of callosal axons. It would be interesting to investigate whether similar cellular strategies are deployed in the spinal cord as well. Analysis of the streams of migrating cells as well as the population of floor plate cells, which is heterogeneous, should be undertaken in the future. Thus, it remains quite difficult at the moment to make a true comparison of the mechanisms controlling midline crossing in the brain and in the spinal cord. Some of them might be conserved. For example, both systems share contribution of attractive and repulsive signaling, relying on the same sets of cues. Among the potential differences is the presence of pioneers for callosal axons, which has not been reported in the spinal cord and the contribution of guideposts including transient neuronal populations at the cortical midline, which have not been described in the spinal floor plate. In all cases, detailed characterization of the distribution of the guidance cues at the midline and of the dynamics of guidance receptors would greatly facilitate our current understanding of the mechanisms of midline crossing and the specificity that may exist in the brain and in the spinal cord.

Finally, new links between neuro-developmental diseases and axon navigation emerged from the characterization of human mutations in genes encoding the guidance machinery. Moreover, this also facilitated the identification of effectors of commissure formation. Likewise, mutations causing midline crossing defects and resulting in functional deficits have been found in the genes encoding Robo3 and DCC [6]. Missense and splice-site mutations in genes encoding $\alpha$ - and $\beta$-tubulin isotypes have also been found responsible for complex malformation of the cerebral cortex and corpus callosum agenesis [148]. Significant advances in our current knowledge of midline crossing are expected from the progress in the identification of pathogenic mutations in humans.

\section{Acknowledgment}

The author acknowledges Ed Derrington for the reading and helpful suggestions on the paper.

\section{References}

[1] S. Vulliemoz, O. Raineteau, and D. Jabaudon, "Reaching beyond the midline: why are human brains cross wired?" The Lancet Neurology, vol. 4, no. 2, pp. 87-99, 2005.

[2] R. M. Fame, J. L. MacDonald, and J. D. Macklis, "Development, specification, and diversity of callosal projection neurons," Trends in Neurosciences, vol. 34, no. 1, pp. 41-50, 2011.

[3] A. Fitsiori, D. Nguyen, A. Karentzos, J. Delavelle, and M. I. Vargas, "The corpus callosum: white matter or terra incognita," British Journal of Radiology, vol. 84, no. 997, pp. 5-18, 2011.

[4] D. Kamnasaran, "Agenesis of the corpus callosum: lessons from humans and mice," Clinical and Investigative Medicine, vol. 28, no. 5, pp. 267-282, 2005.

[5] S. Kenwrick, A. Watkins, and E. De Angelis, "Neural cell recognition molecule L1: relating biological complexity to human disease mutations," Human Molecular Genetics, vol. 9, no. 6, pp. 879-886, 2000.

[6] E. C. Engle, "Human genetic disorders of axon guidance," Cold Spring Harbor Perspectives in Biology, vol. 2, no. 3, Article ID a001784, 2010. 
[7] K. Saxena, L. Tamm, A. Walley et al., "A preliminary investigation of corpus callosum and anterior commissure aberrations in aggressive youth with bipolar disorders," Journal of Child and Adolescent Psychopharmacology, vol. 22, pp. 112-119, 2012.

[8] H. Choi, M. Kubicki, T. J. Whitford et al., "Diffusion tensor imaging of anterior commissural fibers in patients with schizophrenia," Schizophrenia Research, vol. 130, no. 1-3, pp. 78-85, 2011.

[9] R. M. Shuman, "The Chiari malformations: a constellation of anomalies," Seminars in Pediatric Neurology, vol. 2, no. 3, pp. 220-226, 1995.

[10] C. Herweh, M. Akbar, M. Wengenroth et al., "DTI of commissural fibers in patients with Chiari II-malformation," NeuroImage, vol. 44, no. 2, pp. 306-311, 2009.

[11] M. Hayashi, K. Sakamoto, K. Kurata, J. Nagata, J. Satoh, and Y. Morimatsu, "Septo-optic dysplasia with cerebellar hypoplasia in Cornelia de Lange syndrome," Acta Neuropathologica, vol. 92, no. 6, pp. 625-630, 1996.

[12] D. M. Clark, I. Sherer, M. A. Deardorff et al., "Identification of a prenatal profile of Cornelia de Lange Syndrome (CdLS): a review of 53 CdLS pregnancies," American Journal of Medical Genetics A, vol. 158, pp. 1848-1856, 2012.

[13] M. A. Tischfield, H. N. Baris, C. Wu et al., "Human TUBB3 mutations perturb microtubule dynamics, kinesin interactions, and axon guidance," Cell, vol. 140, no. 1, pp. 74-87, 2010.

[14] M. Hingorani, I. Hanson, and V. van Heyningen, "Aniridia," European Journal of Human Genetics, vol. 20, pp. 1011-1017, 2012.

[15] D. E. Bamiou, S. L. Free, S. M. Sisodiya et al., "Auditory interhemispheric transfer deficits, hearing difficulties, and brain magnetic resonance imaging abnormalities in children with congenital aniridia due to PAX6 mutations," Archives of Pediatrics and Adolescent Medicine, vol. 161, no. 5, pp. 463-469, 2007.

[16] G. B. Northam, F. Liégeois, J. D. Tournier et al., "Interhemispheric temporal lobe connectivity predicts language impairment in adolescents born preterm," Brain, vol. 135, pp. 37813798, 2012.

[17] G. J. Bashaw and R. Klein, "Signaling from axon guidance receptors," Cold Spring Harbor Perspectives in Biology, vol. 2, no. 5, Article ID a001941, 2010.

[18] J. Raper and C. Mason, "Cellular strategies of axonal pathfinding," Cold Spring Harbor Perspectives in Biology, vol. 2, no. 9, Article ID a001933, 2010.

[19] E. A. Vitriol and J. Q. Zheng, "Growth cone travel in space and time: the cellular ensemble of cytoskeleton, adhesion, and membrane," Neuron, vol. 73, pp. 1068-1081, 2012.

[20] V. V. Chizhikov and K. J. Millen, "Roof plate-dependent patterning of the vertebrate dorsal central nervous system," Developmental Biology, vol. 277, no. 2, pp. 287-295, 2005.

[21] N. A. Bermingham, B. A. Hassan, V. Y. Wang et al., "Proprioceptor pathway development is dependent on MATH1," Neuron, vol. 30, no. 2, pp. 411-422, 2001.

[22] K. J. Lee, M. Mendelsohn, and T. M. Jessell, "Neuronal patterning by BMPs: a requirement for GDF7 in the generation of a discrete class of commissural interneurons in the mouse spinal cord," Genes and Development, vol. 12, no. 21, pp. 3394-3407, 1998.

[23] Y. Muroyama, M. Fujihara, M. Ikeya, H. Kondoh, and S. Takada, "Wnt signaling plays an essential role in neuronal specification of the dorsal spinal cord," Genes and Development, vol. 16, no. 5, pp. 548-553, 2002.
[24] K. F. Liem Jr., G. Tremml, and T. M. Jessell, "A role for the roof plate and its resident TGF $\beta$-related proteins in neuronal patterning in the dorsal spinal cord," Cell, vol. 91, no. 1, pp. 127$138,1997$.

[25] S. I. Wilson, B. Shafer, K. J. Lee, and J. Dodd, "A molecular program for contralateral trajectory: Rig-1 control by LIM homeodomain transcription factors," Neuron, vol. 59, no. 3, pp. 413424, 2008.

[26] Q. Ding, P. S. Joshi, Z. H. Xie, M. Xiang, and L. Gan, "BARHL2 transcription factor regulates the ipsilateral/contralateral subtype divergence in postmitotic dI1 neurons of the developing spinal cord," Proceedings of the National Academy of Sciences of the United States of America, vol. 109, pp. 1566-1571, 2012.

[27] T. J. Petros, A. Rebsam, and C. A. Mason, "Retinal axon growth at the optic chiasm: to cross or not to cross," Annual Review of Neuroscience, vol. 31, pp. 295-315, 2008.

[28] A. Augsburger, A. Schuchardt, S. Hoskins, J. Dodd, and S. Butler, "Bmps as mediators of roof plate repulsion of commissural neurons," Neuron, vol. 24, no. 1, pp. 127-141, 1999.

[29] K. Yamauchi, S. G. Varadarajan, J. E. Li, and S. J. Butler, "Type Ib BMP receptors mediate the rate of commissural axon extension through inhibition of cofilin activity," Development, vol. 140, pp. 333-342, 2013.

[30] D. Bagnard, N. Thomasset, M. Lohrum, A. W. Püschel, and J. Bolz, "Spatial distributions of guidance molecules regulate chemorepulsion and chemoattraction of growth cones," The Journal of Neuroscience, vol. 20, no. 3, pp. 1030-1035, 2000.

[31] S. M. Islam, Y. Shinmyo, T. Okafuji et al., "Draxin, a repulsive guidance protein for spinal cord and forebrain commissures," Science, vol. 323, no. 5912, pp. 388-393, 2009.

[32] T. Serafini, T. E. Kennedy, M. J. Galko, C. Mirzayan, T. M. Jessell, and M. Tessier- Lavigne, "The netrins define a family of axon outgrowth-promoting proteins homologous to C. elegans UNC-6," Cell, vol. 78, no. 3, pp. 409-424, 1994.

[33] T. Serafini, S. A. Colamarino, E. D. Leonardo et al., "Netrin-1 is required for commissural axon guidance in the developing vertebrate nervous system," Cell, vol. 87, no. 6, pp. 1001-1014, 1996.

[34] A. Fazeli, S. L. Dickinson, M. L. Hermiston et al., "Phenotype of mice lacking functional Deleted in colorectal cancer (Dcc) gene," Nature, vol. 386, no. 6627, pp. 796-804, 1997.

[35] T. E. Kennedy, H. Wang, W. Marshall, and M. Tessier-Lavigne, "Axon guidance by diffusible chemoattractants: a gradient of netrin protein in the developing spinal cord," The Journal of Neuroscience, vol. 26, no. 34, pp. 8866-8874, 2006.

[36] M. Brankatschk and B. J. Dickson, "Netrins guide Drosophila commissural axons at short range," Nature Neuroscience, vol. 9, no. 2, pp. 188-194, 2006.

[37] R. Harris, L. M. Sabatelli, and M. A. Seeger, "Guidance cues at the Drosophila CNS midline: identification and characterization of two Drosophila Netrin/UNC-6 homologs," Neuron, vol. 17, no. 2, pp. 217-228, 1996.

[38] A. Ly, A. Nikolaev, G. Suresh, Y. Zheng, M. Tessier-Lavigne, and E. Stein, "DSCAM is a netrin receptor that collaborates with DCC in mediating turning responses to Netrin-1," Cell, vol. 133, no. 7, pp. 1241-1254, 2008.

[39] G. Liu, W. Li, L. Wang et al., "DSCAM functions as a netrin receptor in commissural axon pathfinding," Proceedings of the National Academy of Sciences of the United States of America, vol. 106, no. 8, pp. 2951-2956, 2009. 
[40] E. Palmesino, P. C. Haddick, M. Tessier-Lavigne, and A. Kania, "Genetic analysis of DSCAM's role as a Netrin-1 receptor in vertebrates," The Journal of Neuroscience, vol. 32, pp. 411-416, 2012.

[41] V. Corset, K. T. Nguyen-Ba-Charvet, C. Forcet, E. Moyse, A. Chédotal, and P. Mehlen, "Netrin-1-mediated axon outgrowth and CAMP production requires interaction with adenosine $\mathrm{A} 2 \mathrm{~b}$ receptor," Nature, vol. 407, no. 6805, pp. 747-750, 2000.

[42] W. L. McKenna, C. Wong-Staal, G. C. Kim, H. Macias, L. Hinck, and J. L. Bartoe, "Netrin-1-independent adenosine A2b receptor activation regulates the response of axons to Netrin-1 by controlling cell surface levels of UNC5A receptors," Journal of Neurochemistry, vol. 104, no. 4, pp. 1081-1090, 2008.

[43] N. Rama, D. Goldschneider, V. Corset, J. Lambert, L. Pays, and P. Mehlen, "Amyloid precursor protein regulates Netrin-1mediated commissural axon outgrowth," The Journal of Biological Chemistry, vol. 287, pp. 30014-30023, 2012.

[44] F. Charron, E. Stein, J. Jeong, A. P. McMahon, and M. Tessier-Lavigne, "The morphogen sonic hedgehog is an axonal chemoattractant that collaborates with Netrin-1 in midline axon guidance," Cell, vol. 113, no. 1, pp. 11-23, 2003.

[45] A. Okada, F. Charron, S. Morin et al., "Boc is a receptor for sonic hedgehog in the guidance of commissural axons," Nature, vol. 444, no. 7117, pp. 369-373, 2006.

[46] C. Ruiz de Almodovar, P. J. Fabre, E. Knevels et al., "VEGF mediates commissural axon chemoattraction through its receptor Flk1," Neuron, vol. 70, no. 5, pp. 966-978, 2011.

[47] C. Kiecker and A. Lumsden, "The role of organizers in patterning the nervous system," Annual Review of Neuroscience, vol. 35, pp. 347-367, 2012.

[48] G. Le Dreau and E. Marti, "Dorsal-ventral patterning of the neural tube: a tale of three signals," Developmental Neurobiology, vol. 72, pp. 1471-1481, 2012.

[49] M. Joksimovic, M. Patel, M. M. Taketo, R. Johnson, and R. Awatramani, "Ectopic Wnt/beta-catenin signaling induces neurogenesis in the spinal cord and hindbrain floor plate," PloS One, vol. 7, Article ID e30266, 2012.

[50] H. Nawabi and V. Castellani, "Axonal commissures in the central nervous system: how to cross the midline?" Cellular and Molecular Life Sciences, vol. 68, no. 15, pp. 2539-2553, 2011.

[51] H. Nawabi, A. Briançon-Marjollet, C. Clark et al., "A midline switch of receptor processing regulates commissural axon guidance in vertebrates," Genes and Development, vol. 24, no. 4, pp. 396-410, 2010.

[52] C. Charoy, H. Nawabi, F. Reynaud et al., "gdnf activates midline repulsion by Semaphorin3B via NCAM during commissural axon guidance," Neuron, vol. 75, pp. 1051-1066, 2012.

[53] D. L. Black and S. L. Zipursky, “To cross or not to cross: alternatively spliced forms of the robo3 receptor regulate discrete steps in axonal midline crossing," Neuron, vol. 58, no. 3, pp. 297-298, 2008.

[54] B. J. Dickson and G. F. Gilestro, "Regulation of commissural axon pathfinding by Slit and its Robo receptors," Annual Review of Cell and Developmental Biology, vol. 22, pp. 651-675, 2006.

[55] T. A. Evans and G. J. Bashaw, "Axon guidance at the midline: of mice and flies," Current Opinion in Neurobiology, vol. 20, no. 1, pp. 79-85, 2010.

[56] R. Shirasaki, R. Katsumata, and F. Murakami, "Change in chemoattractant responsiveness of developing axons at an intermediate target," Science, vol. 279, no. 5347, pp. 105-107, 1998.
[57] J. L. Bonkowsky, S. Yoshikawa, D. D. O’Keefe, A. L. Scully, and J. B. Thomas, "Axon routing across the midline controlled by the Drosophila Derailed receptor," Nature, vol. 402, no. 6761, pp. 540-544, 1999.

[58] T. Kidd, C. Russell, C. S. Goodman, and G. Tear, "Dosage-sensitive and complementary functions of roundabout and commissureless control axon crossing of the CNS midline," Neuron, vol. 20, no. 1, pp. 25-33, 1998.

[59] T. Kidd, K. S. Bland, and C. S. Goodman, "Slit is the midline repellent for the Robo receptor in Drosophila," Cell, vol. 96, no. 6, pp. 785-794, 1999.

[60] M. Georgiou and G. Tear, "Commissureless is required both in commissural neurones and midline cells for axon guidance across the midline," Development, vol.129, no. 12, pp. 2947-2956, 2002.

[61] M. Georgiou and G. Tear, "The N-terminal and transmembrane domains of Commissureless are necessary for its function and trafficking within neurons," Mechanisms of Development, vol. 120, no. 9, pp. 1009-1019, 2003.

[62] K. Keleman, S. Rajagopalan, D. Cleppien et al., "Comm sorts Robo to control axon guidance at the Drosophila midline," Cell, vol. 110, no. 4, pp. 415-427, 2002.

[63] K. Keleman, C. Ribeiro, and B. J. Dickson, "Comm function in commissural axon guidance: cell-autonomous sorting of Robo in vivo," Nature Neuroscience, vol. 8, no. 2, pp. 156-163, 2005.

[64] K. Brose, K. S. Bland, H. W. Kuan et al., "Slit proteins bind robo receptors and have an evolutionarily conserved role in repulsive axon guidance," Cell, vol. 96, no. 6, pp. 795-806, 1999.

[65] T. Kidd, K. Brose, K. J. Mitchell et al., "Roundabout controls axon crossing of the CNS midline and defines a novel subfamily of evolutionarily conserved guidance receptors," Cell, vol. 92, no. 2, pp. 205-215, 1998.

[66] G. F. Gilestro, "Redundant mechanisms for regulation of midline crossing in Drosophila," PLoS ONE, vol. 3, no. 11, Article ID e3798, 2008.

[67] B. Spitzweck, M. Brankatschk, and B. J. Dickson, "Distinct protein domains and expression patterns confer divergent axon guidance functions for Drosophila robo Receptors," Cell, vol. 140, no. 3, pp. 409-420, 2010.

[68] L. Yang, D. S. Garbe, and G. J. Bashaw, "A frazzled/DCCdependent transcriptional switch regulates midline axon guidance," Science, vol. 324, no. 5929, pp. 944-947, 2009.

[69] Q. X. Liu, M. Hiramoto, H. Ueda, T. Gojobori, Y. Hiromi, and S. Hirose, "Midline governs axon pathfinding by coordinating expression of two major guidance systems," Genes and Development, vol. 23, no. 10, pp. 1165-1170, 2009.

[70] H. Long, C. Sabatier, L. Ma et al., "Conserved roles for Slit and Robo proteins in midline commissural axon guidance," Neuron, vol. 42, no. 2, pp. 213-223, 2004.

[71] Y. Zou, E. Stoeckli, H. Chen, and M. Tessier-Lavigne, "Squeezing axons out of the gray matter: a role for slit and Semaphorin proteins from midline and ventral spinal cord," Cell, vol. 102, no. 3, pp. 363-375, 2000.

[72] Z. Chen, B. B. Gore, H. Long, L. Ma, and M. Tessier-Lavigne, "Alternative splicing of the Robo3 axon guidance receptor governs the midline switch from attraction to repulsion," Neuron, vol. 58, no. 3, pp. 325-332, 2008.

[73] C. Sabatier, A. S. Plump, L. Ma et al., "The divergent robo family protein Rig-1/Robo3 is a negative regulator of slit responsiveness required for midline crossing by commissural axons," Cell, vol. 117, no. 2, pp. 157-169, 2004. 
[74] H. A. Coleman, J. P. Labrador, R. K. Chance, and G. J. Bashaw, "The Adam family metalloprotease Kuzbanian regulates the cleavage of the roundabout receptor to control axon repulsion at the midline," Development, vol. 137, no. 14, pp. 2417-2426, 2010.

[75] J. Yuasa-Kawada, M. Kinoshita-Kawada, G. Wu, Y. Rao, and J. Y. $\mathrm{Wu}$, "Midline crossing and Slit responsiveness of commissural axons require USP33," Nature Neuroscience, vol. 12, no. 9, pp. 1087-1089, 2009.

[76] M. Philipp, V. Niederkofler, M. Debrunner, T. Alther, B. Kunz, and E. T. Stoeckli, "RabGDI controls axonal midline crossing by regulating Robol surface expression," Neural Development, vol. 7, article 36, 2012.

[77] K. I. Kuwako, K. Kakumoto, T. Imai et al., "Neural RNA-binding protein musashil controls midline crossing of precerebellar neurons through posttranscriptional regulation of Robo3/Rig-1 expression," Neuron, vol. 67, no. 3, pp. 407-421, 2010.

[78] D. Yan and X. Lin, "Shaping morphogen gradients by proteoglycans," Cold Spring Harbor Perspectives in Biology, vol. 1, no. 3, Article ID a002493, 2009.

[79] A. Gallet, L. Staccini-Lavenant, and P. P. Thérond, "Cellular trafficking of the glypican dally-like is required for full-strength hedgehog signaling and wingless transcytosis," Developmental Cell, vol. 14, no. 5, pp. 712-725, 2008.

[80] H. Hu, "Cell-surface heparan sulfate is involved in the repulsive guidance activities of Slit2 protein," Nature Neuroscience, vol. 4, no. 7, pp. 695-701, 2001.

[81] K. G. Johnson, A. Ghose, E. Epstein, J. Lincecum, M. B. O'Connor, and D. Van Vactor, "Axonal heparan sulfate proteoglycans regulate the distribution and efficiency of the repellent slit during midline axon guidance," Current Biology, vol. 14, no. 6, pp. 499-504, 2004.

[82] T. Xiao, W. Staub, E. Robles, N. J. Gosse, G. J. Cole, and H. Baier, "Assembly of lamina-specific neuronal connections by slit bound to type IV collagen," Cell, vol. 146, no. 1, pp. 164-176, 2011.

[83] K. M. Wright, K. A. Lyon, H. Leung, D. J. Leahy, L. Ma, and D. D. Ginty, "Dystroglycan organizes axon guidance cue localization and axonal pathfinding," Neuron, vol. 76, pp. 931-944, 2012.

[84] N. Yokoyama, M. I. Romero, C. A. Cowan et al., "Forward signaling mediated by ephrin-B3 prevents contralateral corticospinal axons from recrossing the spinal cord midline," Neuron, vol. 29, no. 1, pp. 85-97, 2001.

[85] K. Kullander, N. K. Mather, F. Diella, M. Dottori, A. W. Boyd, and R. Klein, "Kinase-dependent and kinase-independent functions of EphA4 receptors in major axon tract formation in vivo," Neuron, vol. 29, no. 1, pp. 73-84, 2001.

[86] R. Lee, T. J. Petros, and C. A. Mason, "Zic2 regulates retinal ganglion cell axon avoidance of ephrinB2 through inducing expression of the guidance receptor EphB1," The Journal of Neuroscience, vol. 28, no. 23, pp. 5910-5919, 2008.

[87] S. R. Kadison, T. Mäkinen, R. Klein, M. Henkemeyer, and Z. Kaprielian, "EphB receptors and ephrin-B3 regulate axon guidance at the ventral midline of the embryonic mouse spinal cord," The Journal of Neuroscience, vol. 26, no. 35, pp. 8909-8914, 2006.

[88] R. P. Kruger, J. Aurandt, and K. L. Guan, "Semaphorins command cells to move," Nature Reviews Molecular Cell Biology, vol. 6, no. 10, pp. 789-800, 2005.

[89] V. Castellani, "The function of neuropilin/L1 complex," Advances in Experimental Medicine and Biology, vol. 515, pp. 91-102, 2002.
[90] L. M. Parra and Y. Zou, "Sonic hedgehog induces response of commissural axons to Semaphorin repulsion during midline crossing," Nature Neuroscience, vol. 13, no. 1, pp. 29-35, 2010.

[91] E. Stein and M. Tessier-Lavigne, "Hierarchical organization of guidance receptors: silencing of netrin attraction by slit through a Robo/DCC receptor complex," Science, vol. 291, no. 5510, pp. 1928-1938, 2001.

[92] B. B. Gore, K. G. Wong, and M. Tessier-Lavigne, "Stem cell factor functions as an outgrowth-promoting factor to enable axon exit from the midline intermediate target," Neuron, vol. 57, no. 4, pp. 501-510, 2008.

[93] D. Bourikas, V. Pekarik, T. Baeriswyl et al., "Sonic hedgehod guides commissural axons along the longitudinal axis of the spinal cord," Nature Neuroscience, vol. 8, no. 3, pp. 297-304, 2005.

[94] P. T. Yam, C. B. Kent, S. Morin et al., "14-3-3 proteins regulate a cell-intrinsic switch from sonic hedgehog-mediated commissural axon attraction to repulsion after midline crossing," Neuron, vol. 76, pp. 735-749, 2012.

[95] M. L. Baudet, K. H. Zivraj, C. Abreu-Goodger et al., "miR-124 acts through CoREST to control onset of Sema3A sensitivity in navigating retinal growth cones," Nature Neuroscience, vol. 15, pp. 29-38, 2012.

[96] E. T. Stoeckli and L. T. Landmesser, "Axonin-1, Nr-CAM, and $\mathrm{Ng}$-CAM play different roles in the in vivo guidance of chick commissural neurons," Neuron, vol. 14, no. 6, pp. 1165-1179, 1995.

[97] E. T. Stoeckli, P. Sonderegger, G. E. Pollerberg, and L. T. Landmesser, "Interference with axonin-1 and NrCAM interactions unmasks a floor-plate activity inhibitory for commissural axons," Neuron, vol. 18, no. 2, pp. 209-221, 1997.

[98] T. Burstyn-Cohen, V. Tzarfaty, A. Frumkin, Y. Feinstein, E. Stoeckli, and A. Klar, "F-spondin is required for accurate pathfinding of commissural axons at the floor plate," Neuron, vol. 23, no. 2, pp. 233-246, 1999.

[99] R. Imondi, A. R. Jevince, A. W. Helms, J. E. Johnson, and Z. Kaprielian, "Mis-expression of L1 on pre-crossing spinal commissural axons disrupts pathfinding at the ventral midline," Molecular and Cellular Neuroscience, vol. 36, no. 4, pp. 462-471, 2007.

[100] O. Avraham, L. Vald, S. Zisman, A. Schejter, and A. Visel, “Transcriptional control of axonal guidance and sorting in dorsal interneurons by the Lim-HD proteins Lhx9 and Lhxl," Neural Development, vol. 4, no. 1, article 21, 2009.

[101] A. I. Lyuksyutova, C. C. Lu, N. Milanesio et al., "Anteriorposterior guidance of commissural axons by Wnt-Frizzled signaling," Science, vol. 302, no. 5652, pp. 1984-1988, 2003.

[102] E. Domanitskaya, A. Wacker, O. Mauti et al., "Sonic hedgehog guides post-crossing commissural axons both directly and indirectly by regulating Wnt activity," The Journal of Neuroscience, vol. 30, no. 33, pp. 11167-11176, 2010.

[103] P. Joset, A. Wacker, R. Babey et al., "Rostral growth of commissural axons requires the cell adhesion molecule MDGA2," Neural Development, vol. 6, no. 1, article 22, 2011.

[104] V. Niederkofler, T. Baeriswyl, R. Ott, and E. T. Stoeckli, "Nectinlike molecules/SynCAMs are required for post-crossing commissural axon guidance," Development, vol. 137, no. 3, pp. 427435, 2010.

[105] S. Rajagopalan, E. Nicolas, V. Vivancos, J. Berger, and B. J. Dickson, "Crossing the midline: roles and regulation of Robo receptors," Neuron, vol. 28, no. 3, pp. 767-777, 2000. 
[106] J. H. Simpson, T. Kidd, K. S. Bland, and C. S. Goodman, "Shortrange and long-range guidance by Slit and its robo receptors: Robo and Robo2 play distinct roles in midline guidance," Neuron, vol. 28, no. 3, pp. 753-766, 2000.

[107] B. Spitzweck, M. Brankatschk, and B. J. Dickson, "Distinct protein domains and expression patterns confer divergent axon guidance functions for Drosophila Robo Receptors," Cell, vol. 140, no. 3, pp. 409-420, 2010.

[108] A. Jaworski, H. Long, and M. Tessier-Lavigne, "Collaborative and specialized functions of Robol and Robo2 in spinal commissural axon guidance," The Journal of Neuroscience, vol. 30, no. 28, pp. 9445-9453, 2010.

[109] H. Fujisawa, "From the discovery of neuropilin to the determination of its adhesion sites," Advances in Experimental Medicine and Biology, vol. 515, pp. 1-12, 2002.

[110] D. P. Wolfer, R. J. Giger, M. Stagliar, P. Sonderegger, and H. P. Lipp, "Expression of the axon growth-related neural adhesion molecule TAG-1/axonin-1 in the adult mouse brain," Anatomy and Embryology, vol. 197, no. 3, pp. 177-185, 1998.

[111] E. T. Stoeckli and L. T. Landmesser, "Axon guidance at choice points," Current Opinion in Neurobiology, vol. 8, no. 1, pp. 7379, 1998.

[112] B. Chen, S. S. Wang, A. M. Hattox, H. Rayburn, S. B. Nelson, and S. K. McConnell, "The Fezf2-Ctip2 genetic pathway regulates the fate choice of subcortical projection neurons in the developing cerebral cortex," Proceedings of the National Academy of Sciences of the United States of America, vol. 105, no. 32, pp. 11382-11387, 2008.

[113] E. A. Alcamo, L. Chirivella, M. Dautzenberg et al., "Satb2 regulates callosal projection neuron identity in the developing cerebral cortex," Neuron, vol. 57, no. 3, pp. 364-377, 2008.

[114] C. Baranek, M. Dittrich, S. Parthasarathy et al., "Protooncogene Ski cooperates with the chromatin-remodeling factor Satb2 in specifying callosal neurons," Proceedings of the National Academy of Sciences of the United States of America, vol. 109, pp. 3546-3551, 2012.

[115] R. Hand and F. Polleux, "Neurogenin2 regulates the initial axon guidance of cortical pyramidal neurons projecting medially to the corpus callosum," Neural Development, vol. 6, article 30, 2011.

[116] P. Mattar, O. Britz, C. Johannes et al., "A screen for downstream effectors of Neurogenin 2 in the embryonic neocortex," Developmental Biology, vol. 273, no. 2, pp. 373-389, 2004.

[117] X. Jiang, Y. Zhou, L. Xian, W. Chen, H. Wu, and X. Gao, "The mutation in Chd7 causes misexpression of Bmp4 and developmental defects in telencephalic midline," The American Journal of Pathology, vol. 181, pp. 626-641, 2012.

[118] J. Silver, "Glia-neuron interactions at the midline of the developing mammalian brain and spinal cord," Perspectives on Developmental Neurobiology, vol. 1, no. 4, pp. 227-236, 1993.

[119] C. Lindwall, T. Fothergill, and L. J. Richards, "Commissure formation in the mammalian forebrain," Current Opinion in Neurobiology, vol. 17, no. 1, pp. 3-14, 2007.

[120] M. Piper, R. X. Moldrich, C. Lindwall et al., "Multiple non-cellautonomous defects underlie neocortical callosal dysgenesis in Nfib-deficient mice," Neural Development, vol. 4, no. 1, article 43, 2009.

[121] D. Magnani, K. Hasenpusch-Theil, C. Benadiba et al., "Gli3 controls corpus callosum formation by positioning midline guideposts during telencephalic patterning," Cereb Cortex, 2012.
[122] E. M. Amaniti, K. Hasenpusch-Theil, Z. Li et al., "Gli3 is required in $\mathrm{Emxl}^{+}$progenitors for the development of the corpus callosum," Developmental Biology, vol. 376, pp. 113-124, 2013.

[123] S. Tole, G. Gutin, L. Bhatnagar, R. Remedios, and J. M. Hébert, "Development of midline cell types and commissural axon tracts requires Fgfrl in the cerebrum," Developmental Biology, vol. 289, no. 1, pp. 141-151, 2006.

[124] K. M. Smith, Y. Ohkubo, M. E. Maragnoli et al., "Midline radial glia translocation and corpus callosum formation require FGF signaling," Nature Neuroscience, vol. 9, no. 6, pp. 787-797, 2006.

[125] C. Sánchez-Camacho, J. A. Ortega, I. Ocaña, S. Alcántara, and P. Bovolenta, "Appropriate Bmp7 levels are required for the differentiation of midline guidepost cells involved in corpus callosum formation," Developmental Neurobiology, vol. 71, no. 5, pp. 337-350, 2011.

[126] D. K. Unni, M. Piper, R. X. Moldrich et al., "Multiple Slits regulate the development of midline glial populations and the corpus callosum," Developmental Biology, vol. 365, pp. 36-49, 2012.

[127] W. Hofmeister and B. Key, "Frizzled-3a and Wnt-8b genetically interact during forebrain commissural formation in embryonic zebrafish," Brain Research, vol. 1506, pp. 25-34, 2013.

[128] M. Niquille, S. Garel, F. Mann et al., "Transient neuronal populations are required to guide callosal axons: a role for semaphorin 3C," PLoS Biology, vol. 7, no. 10, Article ID e1000230, 2009.

[129] M. Niquille, S. Minocha, J. P. Hornung et al. et al., "Two specific populations of GABAergic neurons originating from the medial and the caudal ganglionic eminences aid in proper navigation of callosal axons," Developmental Neurobiology, 2013.

[130] C. Benadiba, D. Magnani, M. Niquille et al., "The ciliogenic transcription factor RFX3 regulates early midline distribution of guidepost neurons required for corpus callosum development," PLoS Genetics, vol. 8, Article ID e1002606, 2012.

[131] S. E. Koester and D. D. M. O'Leary, "Axons of early generated neurons in cingulate cortex pioneer the corpus callosum," The Journal of Neuroscience, vol. 14, no. 11, pp. 6608-6620, 1994.

[132] V. Borrell and O. Marín, "Meninges control tangential migration of hem-derived Cajal-Retzius cells via CXCL12/CXCR4 signaling," Nature Neuroscience, vol. 9, no. 10, pp. 1284-1293, 2006.

[133] G. Li, H. Kataoka, S. R. Coughlin, and S. J. Pleasure, "Identification of a transient subpial neurogenic zone in the developing dentate gyrus and its regulation by Cxcl12 and reelin signaling," Development, vol. 136, no. 2, pp. 327-335, 2009.

[134] Y. Choe, J. A. Siegenthaler, and S. J. Pleasure, "A cascade of morphogenic signaling initiated by the meninges controls corpus callosum formation," Neuron, vol. 73, pp. 698-712, 2012.

[135] Y. Wang, J. Zhang, S. Mori, and J. Nathans, "Axonal growth and guidance defects in Frizzled3 knock-out mice: a comparison of diffusion tensor magnetic resonance imaging, neurofilament staining, and genetically directed cell labeling," The Journal of Neuroscience, vol. 26, no. 2, pp. 355-364, 2006.

[136] T. R. Keeble, M. M. Halford, C. Seaman et al., "The Wnt receptor Ryk is required for Wnt5a-mediated axon guidance on the contralateral side of the corpus callosum," The Journal of Neuroscience, vol. 26, no. 21, pp. 5840-5848, 2006.

[137] A. Bagri, O. Marín, A. S. Plump et al., "Slit proteins prevent midline crossing and determine the dorsoventral position of major axonal pathways in the mammalian forebrain," Neuron, vol. 33, no. 2, pp. 233-248, 2002. 
[138] T. Shu, V. Sundaresan, M. M. McCarthy, and L. J. Richards, "Slit2 guides both precrossing and postcrossing callosal axons at the midline in vivo," The Journal of Neuroscience, vol. 23, no. 22, pp. 8176-8184, 2003.

[139] G. Ahmed, Y. Shinmyo, K. Ohta et al. et al., "Draxin inhibits axonal outgrowth through the netrin receptor DCC," The Journal of Neuroscience, vol. 31, pp. 14018-14023, 2011.

[140] C. Gu, E. R. Rodriguez, D. V. Reimert et al., "Neuropilin-1 conveys semaphorin and VEGF signaling during neural and cardiovascular development," Developmental Cell, vol. 5, no. 1, pp. 45-57, 2003.

[141] R. J. Giger, J. F. Cloutier, A. Sahay et al., "Neuropilin-2 is required in vivo for selective axon guidance responses to secreted semaphorins," Neuron, vol. 25, no. 1, pp. 29-41, 2000.

[142] H. Chen, A. Bagri, J. A. Zupicich et al., "Neuropilin-2 regulates the development of select cranial and sensory nerves and hippocampal mossy fiber projections," Neuron, vol. 25, no. 1, pp. 43-56, 2000.

[143] M. Piper, C. Plachez, O. Zalucki et al., "Neuropilin 1-Sema signaling regulates crossing of cingulate pioneering axons during development of the corpus callosum," Cerebral Cortex, vol. 19, supplement 1, pp. i11-i21, 2009.

[144] H. Kamiguchi, M. L. Hlavin, and V. Lemmon, "Role of L1 in neural development: what the knockouts tell us," Molecular and Cellular Neurosciences, vol. 12, no. 1-2, pp. 48-55, 1998.

[145] T. Ren, J. Zhang, C. Plachez, S. Mori, and L. J. Richards, "Diffusion tensor magnetic resonance imaging and tract-tracing analysis of probst bundle structure in netrinl- and DCCdeficient mice," The Journal of Neuroscience, vol. 27, no. 39, pp. 10345-10349, 2007.

[146] T. Fothergill, A. L. Donahoo, A. Douglass et al., "Netrin-DCC signaling regulates corpus callosum formation through attraction of pioneering axons and by modulating Slit2-mediated repulsion," Cereb Cortex, 2013.

[147] G. López-Bendito, A. Cautinat, J. A. Sánchez et al., “Tangential neuronal migration controls axon guidance: a role for neuregulin-1 in thalamocortical axon navigation," Cell, vol. 125, no. 1, pp. 127-142, 2006.

[148] K. Poirier, Y. Saillour, N. Bahi-Buisson et al., "Mutations in the neuronal $\beta$-tubulin subunit TUBB3 result in malformation of cortical development and neuronal migration defects," Human Molecular Genetics, vol. 19, no. 22, pp. 4462-4473, 2010. 

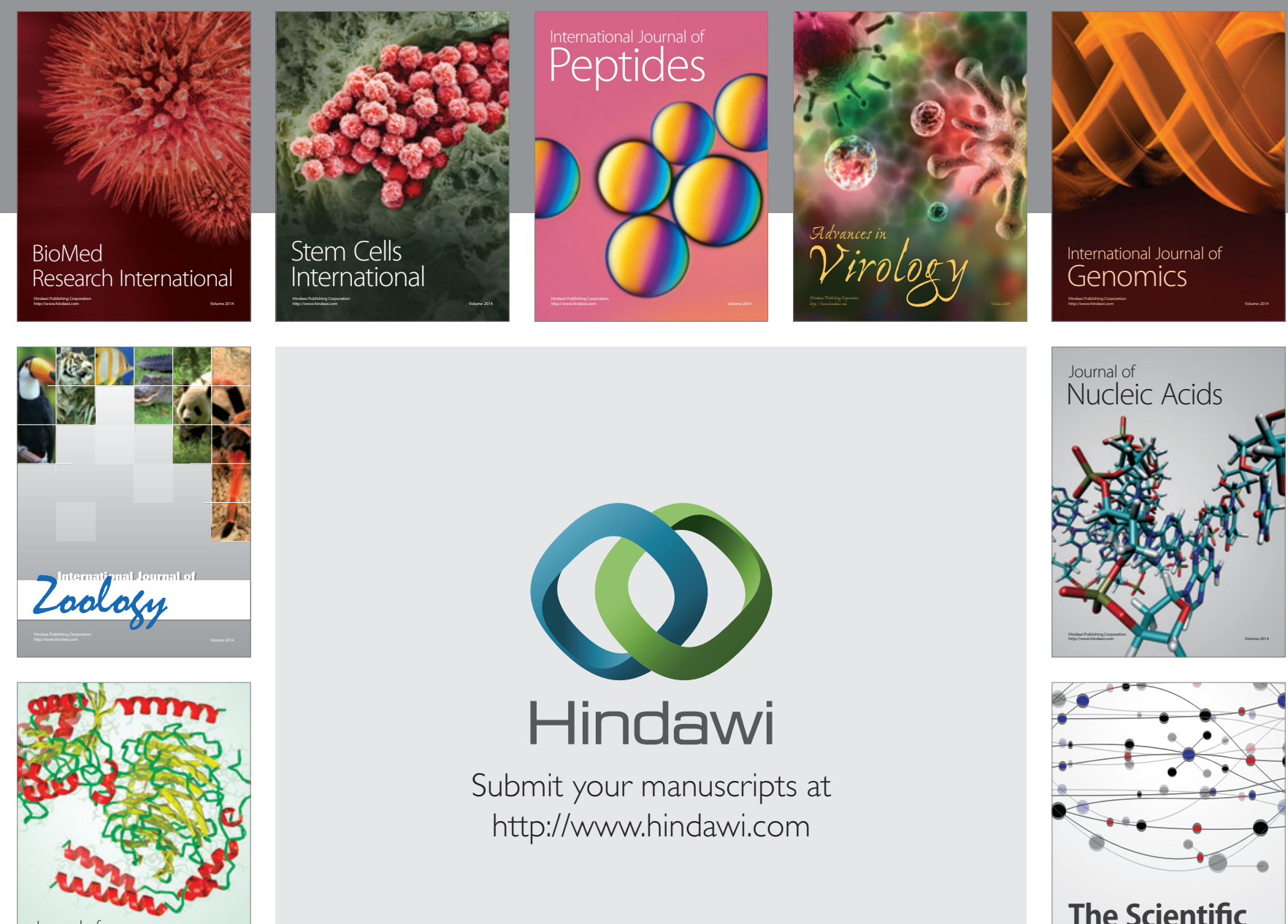

Submit your manuscripts at

http://www.hindawi.com

Journal of
Signal Transduction


The Scientific World Journal
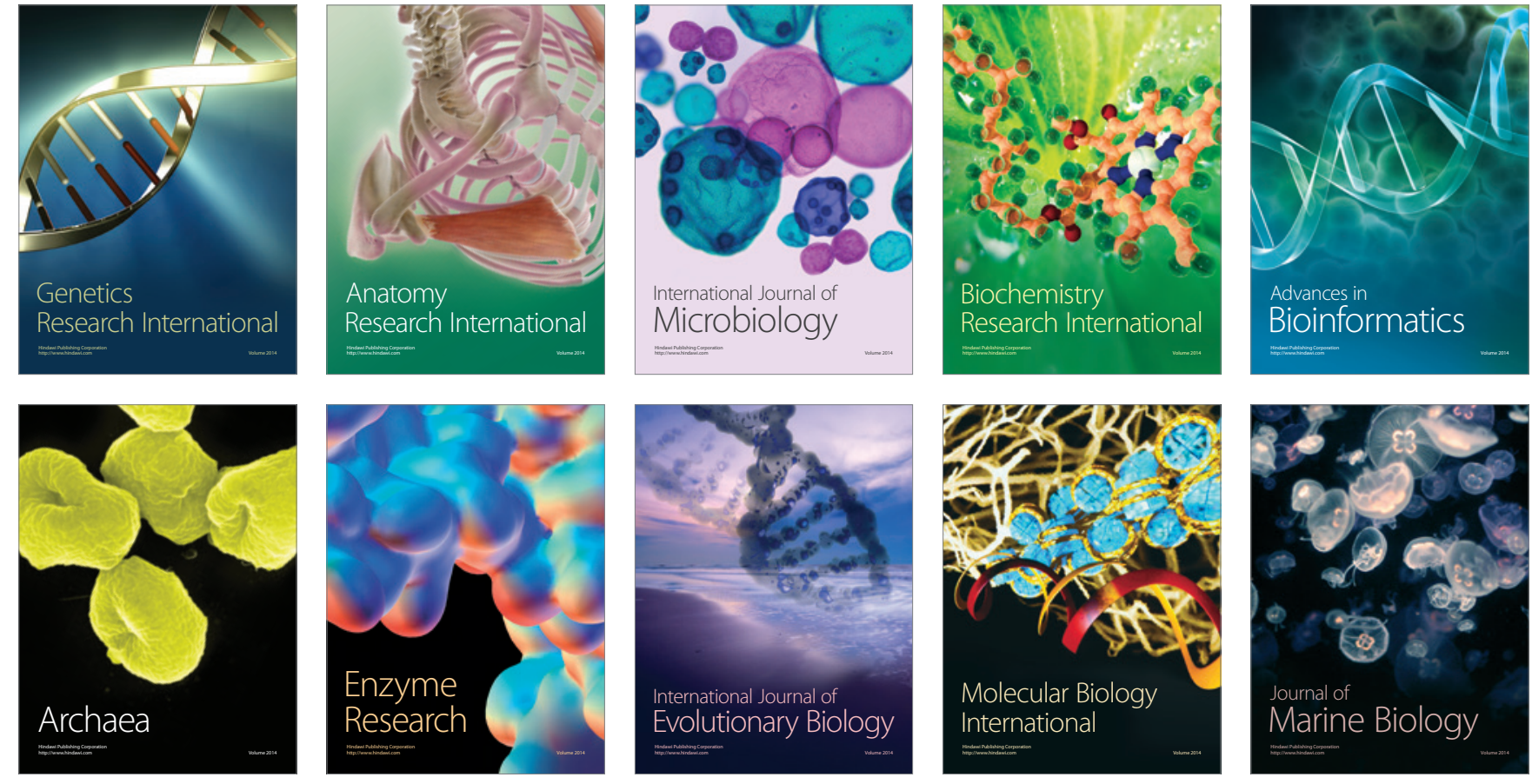